\title{
Environmental enrichment enhances precision and remodeling speed of synaptic nanoarchitecture revealed by crosstalk free two- color in vivo STED nanoscopy
}

\author{
Waja Wegner ${ }^{1,2}$, Heinz Steffens ${ }^{1,2}$, Carola Gregor ${ }^{3}$, Fred Wolf ${ }^{2,4,5}$, Katrin I. Willig ${ }^{1,2,{ }^{*}}$ \\ ${ }^{1}$ Optical Nanoscopy in Neuroscience, Center for Nanoscale Microscopy and Molecular Physiology of the Brain, \\ University Medical Center Göttingen, Göttingen, Germany. \\ ${ }^{2}$ Max Planck Institute of Experimental Medicine, Göttingen, Germany \\ ${ }^{3}$ Department of NanoBiophotonics, Max Planck Institute for Biophysical Chemistry, Göttingen, Germany \\ ${ }^{4}$ Max Planck Institute for Dynamics and Self-Organization, Göttingen, Germany \\ ${ }^{5}$ Campus Institute for Dynamics of Biological Networks, Göttingen, Germany
}

*Corresponding author. Email: kwillig@em.mpg.de

\begin{abstract}
Synaptic plasticity underlies long-lasting structural and functional changes to brain circuitry and its experience-dependent remodeling can be fundamentally enhanced by environmental enrichment. It is unknown, however, whether and how environmental enrichment alters the morphology and dynamics of individual synapses. Here, we present a virtually crosstalk free, two-color in vivo STED microscope to simultaneously superresolve the dynamics of the postsynaptic density protein PSD95 and spine geometry. With environmental enrichment, the size distribution of PSD95 and spine head sizes were narrower than in controls, indicating that synaptic strength is set more precisely with environmental enrichment. Spine head geometry and PSD95 assemblies were highly dynamic exhibiting multiplicative size changes. With environmental enrichment, the topography of PSD95 nanoorganization was more dynamic and the magnitude of reorganization increased systematically with time. Thus, two-color in vivo time-lapse imaging of synaptic nanoorganization uncovers a unique synaptic nanodynamics associated with the enhanced learning capabilities under environmental enrichment.
\end{abstract}




\section{INTRODUCTION}

Excitatory synapses in the brain are mainly formed on dendritic spines and are regarded as the central locus of memory formation. Cognitive, sensory and motor learning can induce specific changes to the cortical circuitry over the entire lifespan. Such experience-dependent plasticity is based on synaptic plasticity, plays a key role for normal brain function and is thought to be neuroprotective against neurodegenerative diseases ${ }^{1,2}$. Memory relies on structures being persistent over long periods of time up to a life-time. There is, however, also evidence that synaptic structure can be highly volatile ${ }^{3,4}$. Synaptic connections thus presumably undergo continuous spontaneous remodeling on top of which activity-dependent changes inscribe memory traces.

Neural activity per se is not required to form an intact synapse; for instance, despite abolishing presynaptic release ${ }^{5}$, or silencing a network, morphologically intact spines are formed ${ }^{6}$. At the beginning of the century, much evidence had shown a strong correlation between the morphological transformation of the spine, termed structural plasticity, and changes in synaptic transmission, termed functional plasticity ${ }^{7}$. However, recent evidence suggests that structural and functional plasticity are not fully interconnected ${ }^{8}$. For example, 'silent' synapses are largely silent at rest but the spine's shape is of mature morphological shape ${ }^{9}$. A main link between structure and function is F-actin, which drives spine morphological remodeling and anchors scaffolding proteins of the post-synaptic density (PSD) such as PSD95. PSD95 in turn anchors amino-3-hydroxy-5-methyl-4-isoxazolepropionic acid (AMPA) receptors via transmembrane AMPAR-regulatory proteins (TARPs) ${ }^{10,11}$ which is the main contribution to synaptic strengthening after long-term potentiation (LTP). Both, anchoring of AMPA receptors and the actin remodeling, are highly dependent on $\mathrm{Ca}^{2+}$ influx after activation, but the intracellular signaling pathways are different as far as known ${ }^{8}$. In addition intrinsic turnover of the cytoskeleton and its different actin pools impose independent contributions to structural remodeling ${ }^{8}$. A plethora of studies show a strong temporal link between AMPA receptor mediated currents and spine volume; both increase rapidly after glutamate uncaging ${ }^{12}$. However, there is recent evidence that not all synaptic proteins increase instantaneously but increase rather with a delay after stimulation. For instance, the number of the scaffolding proteins Homer, Shank and PSD95 at the synapse increases with a delay of $\sim 1$ hour after LTP ${ }^{13,14}$. Thus, the correlation between spine and PSD size which is well documented by electron microscopy ${ }^{14-17}$ may be temporally much less correlated during LTP. These observations suggest that the PSD and spine head plasticity are regulated differentially in the short term and could be more independent than previously thought.

In animal models, experience dependent plasticity is approached by environmental enrichment (EE) which provides the animal with multi-sensory stimulation, cognitive activity, social interactions and physical exercise. Various alterations of brain structure and function by EE have been shown and include increased maturation of new neurons, enhanced expression of signaling molecules and enhanced synaptic plasticity ${ }^{2}$. Structural changes include an increased dendritic branching and spine density in specific cortical regions ${ }^{18,19}$ and an increase in pre- and postsynaptic protein levels in major cortical brain regions ${ }^{20}$. In mouse models of brain diseases strong effects of environmental enrichment are documented ${ }^{2}$. However, non of these studies investigated the ultrastructure or dynamic of the PSD or spine morphology.

Here, we use nanoscale imaging to map temporal changes of the spine head and PSD in vivo and address mainly the following questions. Firstly, how does experience change the synaptic nanoorganization and plasticity? Therefore, we quantified structural correlates as well as the structural plasticity of dendritic spines and PSD95. Secondly, how are fluctuations of the PSD size correlated in time with spine head fluctuations in vivo? For that purpose, we developed a virtually crosstalk free two-color STED microscope to image the spine morphology and PSD95 in vivo at nanoscale resolution. 
This is a significant technical advance over previous two-color live-cell STED microscopy approaches which were performed with a crosstalk of $92 \%$ and $27 \%{ }^{21}$ and therefore required unmixing of the two channels. Hitherto, most in vitro and in vivo studies have been performed using two-photon microscopy to visualize spines and PSDs ${ }^{12-14,16,22-24}$. However, due to its limited optical resolution of 300-500nm it was impossible to quantify precisely spine parameters such as head size and neck length or diameter. Moreover, PSD nanostructures such as perforations ${ }^{25}$ are not detectable with two-photon microscopy. Several studies utilized superresolution microscopy to dissect the synaptic nanoorganization of PSD95 in neuronal cultures or fixed brain slices and showed an arrangement of PSD95 in nanoclusters of 50-250 nm in size ${ }^{26-29}$. Recently, we studied the nanoplasticity of PSD95 with STED microscopy in a PSD95-EGFP transgenic mouse in vivo. We found that PSD95 assemblies were often perforated, formed ring-like or horse-shoe shapes or were clustered; all shapes changed within a few hours ${ }^{30}$. Here, we utilized a transcriptionally regulated antibody-like protein to visualize endogenous PSD95 without introducing overexpression artefacts ${ }^{31}$. Together with a membrane label we recorded temporal morphological changes of the spine head and PSD95 nanoorganization of mice raised in an enriched environment $(\mathrm{EE})$ versus raised in standard cages (Ctr). Effects of environmental enrichment on structure and function have been shown for multiple brain regions ${ }^{2}$. We chose to investigate the synaptic plasticity in the visual cortex since several studies have shown enhanced plasticity of this area in rodents housed in EE cages ${ }^{32,33}$ and it its easily accessible with light microscopy.

Our data show changes in width but not mean value of spine head and PSD95 size distributions following EE. In EE housed mice, PSD95 underwent much stronger directional changes in size as well as in morphology while spine head dynamical changes were unaltered. The changes of PSD95 and spine head size were only mildly correlated and did not show directional change.

\section{RESULTS}

\section{Virtually crosstalk free two-color STED microscope}

To unambiguously dissect the dynamics of distinct synaptic components and examine their interactions crosstalk free nanoscale imaging is of critical importance. We thus established a virtually crosstalk free two-color STED microscope for imaging of EGFP and EYFP or Citrine, respectively. Previous attempts featuring two detection channels were suffering of high crosstalk ${ }^{21}$. In addition to two-color detection, we therefore implemented a two-color excitation to specifically excite the green or yellow fluorescent protein (Fig.1A). This was achieved by adding a pulsed $520 \mathrm{~nm}$ green laser line to the $483 \mathrm{~nm}$ excitation of our previously described in vivo STED microscope ${ }^{34} .520 \mathrm{~nm}$ is at the red edge of the EGFP excitation band and therefore predominantly excites EYFP (or Citrine) (Fig.1B). The STED beam passed a vortex phase plate to create a donut-shaped intensity profile in the focal plane of the objective as previously described ${ }^{35}$. All three beams, two excitation beams and one STED beam, were spatially aligned by dichroic mirrors and the pulses were temporally aligned to overlap in the focal plane. The fluorescence emission was split by a dichroic mirror and detected in two spectrally separated detection channels. EGFP was preferentially detected by detection 1 (Det1, Fig.1B) at 498$510 \mathrm{~nm}$ while EYFP/Citrine emission was detected mostly by detection 2 (Det2, Fig.1B) at $532-555 \mathrm{~nm}$. The 483 and 520nm excitation was switched on and off alternately while recording two consecutive line scans to temporally separate the EGFP and Citrine emission. To determine the crosstalk between both channels, we investigated one-color labelled cells expressing EGFP or EYFP. Live-cell imaging showed that we have designed a virtually crosstalk free microscope with only $~ 8 \%$ crosstalk of EYFP in the EGFP channel and $~ 5 \%$ of the EGFP signal in the EYFP channel (Fig.1C). 


\section{Two-color in vivo STED microscopy of endogenous PSD95 and spine morphology}

Having established the virtually crosstalk free detection of two STED approved fluorescent proteins ${ }^{36,37}$, we set out to simultaneously superresolve the nanoorganization of the post-synaptic density protein PSD95 and the associated spine morphology in vivo. To this end, we generated recombinant adenoassociated viral (AAV) particles expressing fusion proteins under control of the human Synapsin promoter (hSyn). To visualize PSD95, we expressed the transcriptionally regulated antibody-like protein PSD95-FingR that has been shown to label endogenous PSD95 ${ }^{31}$ attached to the fluorescent protein Citrine. To label the membrane, we expressed myr-EGFP-LDLR(Ct), a combination of a myristoylation site (myr), EGFP and the C-terminal (Ct) cytoplasmic domain of low density lipoprotein receptor (LDLR), a potent marker for the dendritic membrane ${ }^{38}$. In order to reduce the density of labelled neurons, the fluorescent labels were incorporated into AAVs in reverse orientation in the vector between double-floxed inverted open reading frames (DIO). Expression of the labels was enabled by Cre recombinase expressing AAVs that were co-injected at low concentration. Hence, in this system the density of labeled neurons is continuously adjustable by the dilution of the Cre expressing AAV. With this set of AAVs we were able to adapt the brightness of the PSD95 and membrane labelling independently of the cell density. Thus, we co-injected the three different AAVs into pyramidal cell layer 5 of the visual cortex. Three to six weeks after transduction, the mice were anaesthetized and a cranial window was inserted over the visual cortex as described before ${ }^{39}$. STED microscopy visualized dendrites, spines, and attached PSD95 assemblies in layer 1 of the visual cortex at superresolution; the images were basically background-free and of high contrast due to the low number of fluorescently labelled neurons (Fig.1C). Due to the low crosstalk, a computational postprocessing such as unmixing was not required. We observed mainly dendrites that expressed both, EGFP and Citrine, and only rarely found dendrites expressing either Citrine or EGFP alone. The inset in Fig.1D reveals a perforated nanoorganization of the endogenous PSD95 that would not be detectable with conventional in vivo microscopy (Fig.1D) due to the insufficient resolution of two-photon microscopy. In general, we obtained a resolution of at least $\sim 70 \mathrm{~nm}$ for Citrine and $\sim 84 \mathrm{~nm}$ for EGFP (Fig.S1A,B).

A

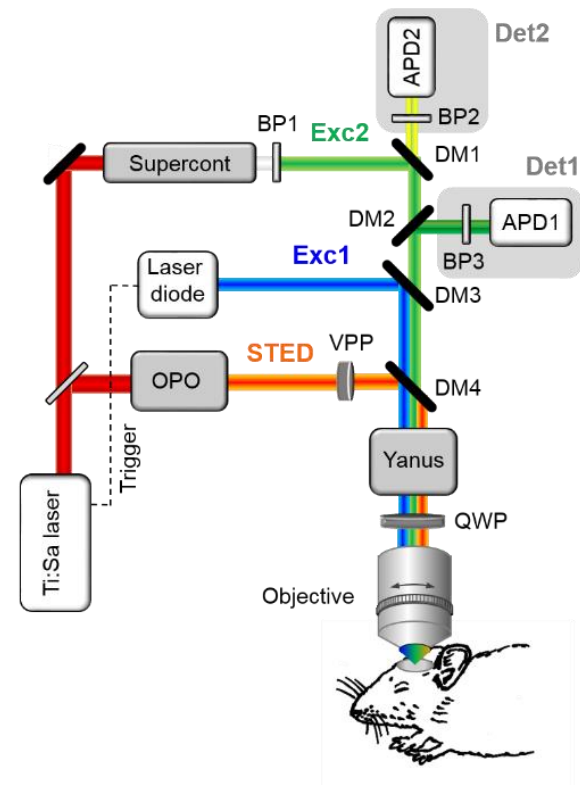

B

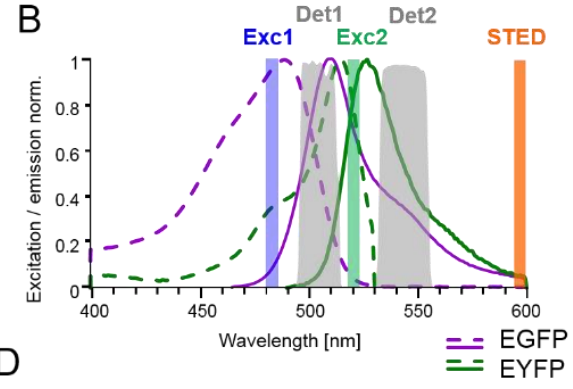

Exc2/Det2

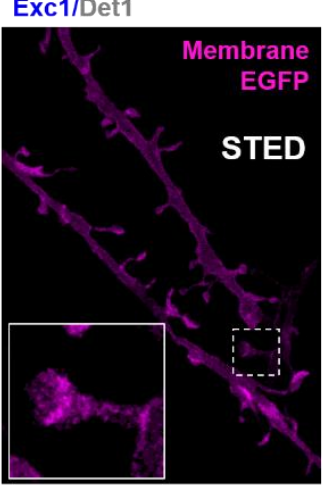

Channel 1 Channel 2 Exc1/Det1 Exc2/Det2
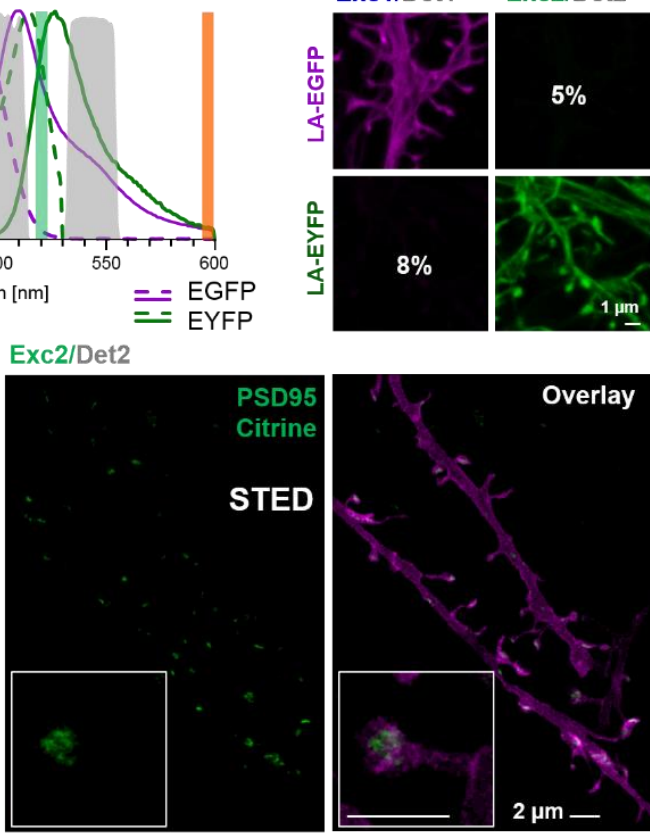

Figure 1: Virtually crosstalk free two-color in vivo STED microscopy. (A) Custom-designed in vivo STED microscope with pulsed $483 \mathrm{~nm}$ (Exc1) and $520 \mathrm{~nm}$ (Exc2) excitation and $595 \mathrm{~nm}$ STED laser. APD: 
Avalanche photon detector, BP: bandpass filter, Det: detection, DM: dichroic mirror, OPO: optical parametric oscillator, QWP: quarter wave plate, VPP: vortex phase plate. Confer Material and Methods section for details. (B) Excitation (dashed line) and emission (solid line) spectrum of EGFP and EYFP and selective excitation and detection. (C) Cultured hippocampal neurons expressing the actin marker Lifeact (LA)-EGFP or LA-EYFP. Excitation of EGFP at $483 \mathrm{~nm}$ close to its excitation maximum (B) and detection at 498-510 $\mathrm{nm}$ (Det1) reduces the crosstalk to 5\% in Channel 2 (C). EYFP or Citrine is excited at $520 \mathrm{~nm}$ close to its maximum (B) and detected at 532-555 nm (Det2) which results in a low crosstalk of $8 \%$ in Channel 1 (C). (D) In vivo STED microscopy image of apical dendrite in layer 1 of the visual cortex of an anaesthetized mouse. Labelling of membrane (myr-EGFP-LDLR(Ct)) and PSD95 (PSD95FingR-Citrine) visualizes the spine morphology at superresolution and PSD95 nanoorganization (D, inset). Images are smoothed and represent maximum intensity projections (MIPs) but without unmixing due to the low crosstalk.

\section{EE housed mice exhibit more sharply defined spine head and PSD95 sizes than Ctr}

Having established the two-color in vivo STED microscope, we addressed the question of whether spine or post-synaptic density exhibit systematic differences between EE and Ctr mice. EE mice were housed in a large, two-floor cage equipped with three running wheels for physical exercise, a labyrinth for cognitive stimulation, a tube and a ladder to change between the two levels, and were kept in groups of up to 12 female mice to allow manifold social interactions (Fig.S2). Control mice were raised in pairs in standard cages without any equipment (Fig.S2). The mice were transduced with AAVs as described above and 3-6 weeks after transduction, a cranial window was implanted above the visual cortex. With the two-color STED microscope, we acquired z-stacks mainly of dendrites, which were in parallel to the focal plane. To analyze differences in morphology of the dendritic spines, we measured the length and width of the spine head and computed the spine head cross sectional area approximating the head by an ellipse (Fig.2A). Analogously, we measured the length and width of the PSD95 assembly and computed the elliptic area (Fig.2A, cf. section Methods for details). We only considered spines containing a PSD95 label and therefore spines, which most likely form functional synapses. We occasionally observed but did not analyze long, thin spines without a head, sometimes called filopodia. We determined a median spine head area for EE housed mice of 0.493 (interquartile range (IR): $0.328-$ 0.813) $\mu \mathrm{m}^{2}$ and 0.477 (IR: 0.297-0.854) $\mu \mathrm{m}^{2}$ for Ctr mice. The area of PSD95 on the same set of spine heads was 0.153 (IR: 0.097-0.255) $\mu \mathrm{m}^{2}$ in median for EE mice and 0.148 (IR: 0.089-0.280) $\mu \mathrm{m}^{2}$ for Ctr mice which is in accordance with previous EM studies in the mouse visual cortex ${ }^{40}$. For comparison, we also determined the brightness of PSD95 assemblies, an often used stand-in for spine size in twophoton microscopy, and found a moderate correlation of $\mathrm{R}^{2}=0.76$ between the brightness and the size determined from our STED data (Fig.S3A). The spine head area correlated with the PSD95 area for EE and Ctr (Fig.2B), corroborating EM and two-photon microscopy studies ${ }^{14-16}$. The histograms of spine head area (Fig.2C) and PSD95 area (Fig.2D) were positively skewed and the distributions were significantly different between EE and Ctr mice. To further dissect these differences, we next plotted the histograms of the logarithm of spine head areas (Fig.2E) and PSD95 areas (Fig.2F). All four histograms (Fig.2E,F) are symmetric and were well described by a Gaussian function. This in line with prior work showing that the spine and PSD95 fluorescence is log-normally distributed in general which is predicted by multiplicative processes of ongoing spine plasticity ${ }^{6,22}$. The Gaussian functions fitting the spine head area (Fig.2E) and PSD95 area (Fig.2F) are almost centered and indeed the average area is not significantly different between EE and Ctr mice (Fig.2G,H). However, we observed a significant difference in variance (Fig.2I,J) visible in the different widths of the Gaussian distributions in Fig.2E,F. Spine density was not significantly different between EE and Ctr housed mice (Fig.S3B). These results 
show that although the average areas of the spine head and PSD95 are similar for EE and Ctr mice, their variance in size is smaller for the enrichment mice than for the control mice, indicating that the synaptic strength are more sharply defined in EE mice.
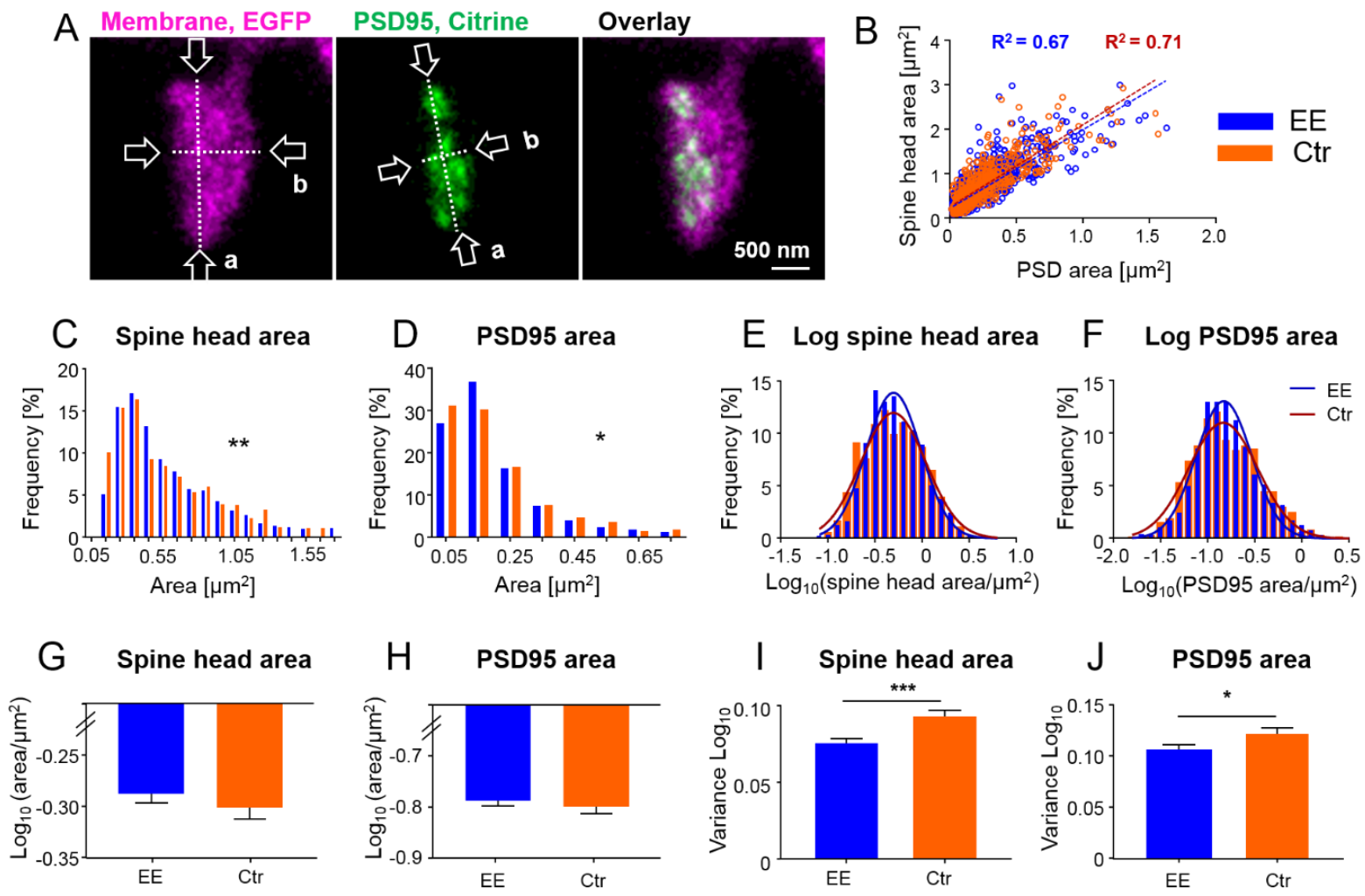

Figure 2: The size distributions of the spine head and PSD95 area are sharper for mice housed in environmentally enriched (EE) than standard (Ctr) cages. (A) STED image of dendritic spine head (left, magenta), associated PSD95 (middle, green) and overlay (right). For both, the length (a) and width (b) were measured to estimate the area. (B) Strong correlation of absolute spine head and PSD95 area in $\mathrm{Ctr}$ (orange) and EE (blue) housed mice. Linear regression lines are dashed and $\mathrm{R}^{2}$ values are displayed (EE and Ctr, deviation from zero: $p<0.0001$ ). (C, D) Frequency distributions of spine head area (C) and PSD95 area (D) are positively skewed and significantly different between EE and Ctr housed mice (Kolmogorov-Smirnov test, $C:{ }^{* *} p=0.009, D:{ }^{*} p=0.039$ ). Graphs display only sizes with a frequency > $1 \%$ and center of BIN. (E, F) Same data as shown in (C, D), plotted on a logarithmic scale. Solid lines represent Gaussian functions fitted to the respective histogram. (G-J) Mean (G: $E_{\text {spine: }}-0.288, \mathrm{Ctr}_{\text {spine }}$ : -0.301, H: EEPSD: -0.787 , CtrPSD: -0.799$)$ and variance $(\mathrm{I}, \mathrm{J})$ of logarithmic data shown in (E, F) + SEM (Unpaired $t$-test with Welch's correction: $\mathrm{G}: \mathrm{p}=0.34, \mathrm{H}: \mathrm{p}=0.47$, I: ${ }^{* *} \mathrm{p}<0.001, \mathrm{~J}:{ }^{*} \mathrm{p}=0.035$ ). Number of analyzed mice and spines: Ctr: 4x \&mice, $\mathrm{n}_{\text {spine }}=737$; EE: 4x \&mice, $\mathrm{n}_{\text {spine }}=1052$.

\section{Correlated changes of PSD95 and spine head area over minutes to hours}

A strong correlation of PSD and spine size has been reported previously and we confirmed it here for the PSD protein PSD95 (Fig.2B). Whether dynamic changes between these two features are also strongly linked, however, has remained unknown. We thus asked whether there were also strong correlations in temporal changes of these parameters. To answer this question, we performed timelapse STED microscopy of spine morphology and PSD95 for EE and Ctr housed mice for up to 4 hours as described above. We recorded STED images at different fields of view (FOVs) for three time points 
at varying time intervals $\Delta \mathrm{t}$ of $30 \mathrm{~min}, 60 \mathrm{~min}$ or $120 \mathrm{~min}$. Figures $3 \mathrm{~A}$ and $\mathrm{B}$ show representative timelapse STED images at a time interval of 30min. The average spine head area and PSD95 area did not change over the time period of $120 \mathrm{~min}$ (Fig.3C). Thus, we did not observe directed changes as a result of light induced stimulation or phototoxicity and neither observed blebbing. We analyzed the spine head and PSD95 area for each time point as described above and computed their normalized size changes over time. Thus, we normalized the changes between two time points to the sum of the area at these time points. With this normalization positive changes (growth) as well as negative changes (shrinkage) are symmetric and have a boundary value of \pm 1 . Scatter plots showed normalized changes of spine head area as a function of the changes of PSD95 area for the different time intervals (Fig.3DF). In all three plots the data points are scattered over all 4 quadrants (for percentage changes refer to Fig. S4A-C). This means that we observed positively correlated changes of growing spine heads and growing PSD95 areas (or shrinking, respectively) (Fig.3G) as well as anti- or negatively correlated changes, i.e. shrinking PSD95 areas on a growing spine or vice versa (Fig. $3 \mathrm{H}$ ). A linear regression revealed a small positive correlation ( $R^{2}$ between 0.02 and 0.11 , Fig.3D-F), such that e.g. an increase of PSD95 area is more frequently accompanied by an increase in spine head area. To further quantify these changes, we asked how strong the changes were and asked whether they changed over time in magnitude. Growing spine heads grew in average by $23-29 \%$ (and shrinking spine heads shrunk by $17-$ 24\%) (Fig.S4D); growth and shrinkage increased slightly with increasing time intervals, but not significantly. PSD95 assemblies grew in average by $22-38 \%$ (Fig. S4E) and shrunk by $25-35 \%$ (Fig.S4E). About half of the spine heads and PSD95 assemblies grew while the other half shrunk (Fig.S4F,G). To sum up, spine heads and PSD95 assemblies were highly dynamic and grew in average by $>22 \%$ and shrunk by $>17 \%$ already over $30 \mathrm{~min}$. These average changes, however, were not significantly different for EE and Ctr and did not increase significantly with increasing time interval.

We next assessed whether the spread of the temporal changes would be different, similar to what we have observed for the distribution of sizes. To this end, we examined the variance of our parameters and computed the total variance for spine head and PSD95 area changes for the different time intervals (Fig.S4H). A significant increase in variance was observed for EE housed mice after 30min while the increase after 120 min was just not significant (Fig.S4H). We separately tested whether this difference was more prominent when computing the variance for correlated and anti-correlated changes separately. To this end, we performed a principal component analysis (PCA) for all three time intervals. Principal component 1 (PC1) is by definition the axis along which the data shows the maximum of variance and the eigenvalue of PC1 is the variance along this axis. The PC1 vector was for all three time intervals in the first (and third) quadrant (Fig.3D-F) and therefore attributing for positive correlation. The variance of PC1 was slightly higher for EE mice at all three time points with a significant increase for the 120min time interval (Fig.S4I). The second principal component (PC2) is by definition perpendicular to PC1 and therefore in the second (and fourth) quadrant (Fig.3D-F). Thus, PC2 quantifies negatively correlated changes in spine head and PSD95 area (Fig.S4J). Interestingly, the variance along PC2 was relatively high ( $30-40 \%$ of total, Fig.S4K), quantifying that a negative correlation of the size changes of PSD95 assemblies and spine head is not a rare phenomenon. In total, these results start to suggest a slightly higher temporal dynamics in EE mice due to the higher total variance for two time-points and higher PC1 value for EE mice. However, the average changes did not differ, neither over time nor between $\mathrm{EE}$ and $\mathrm{Ctr}$ housed mice. 

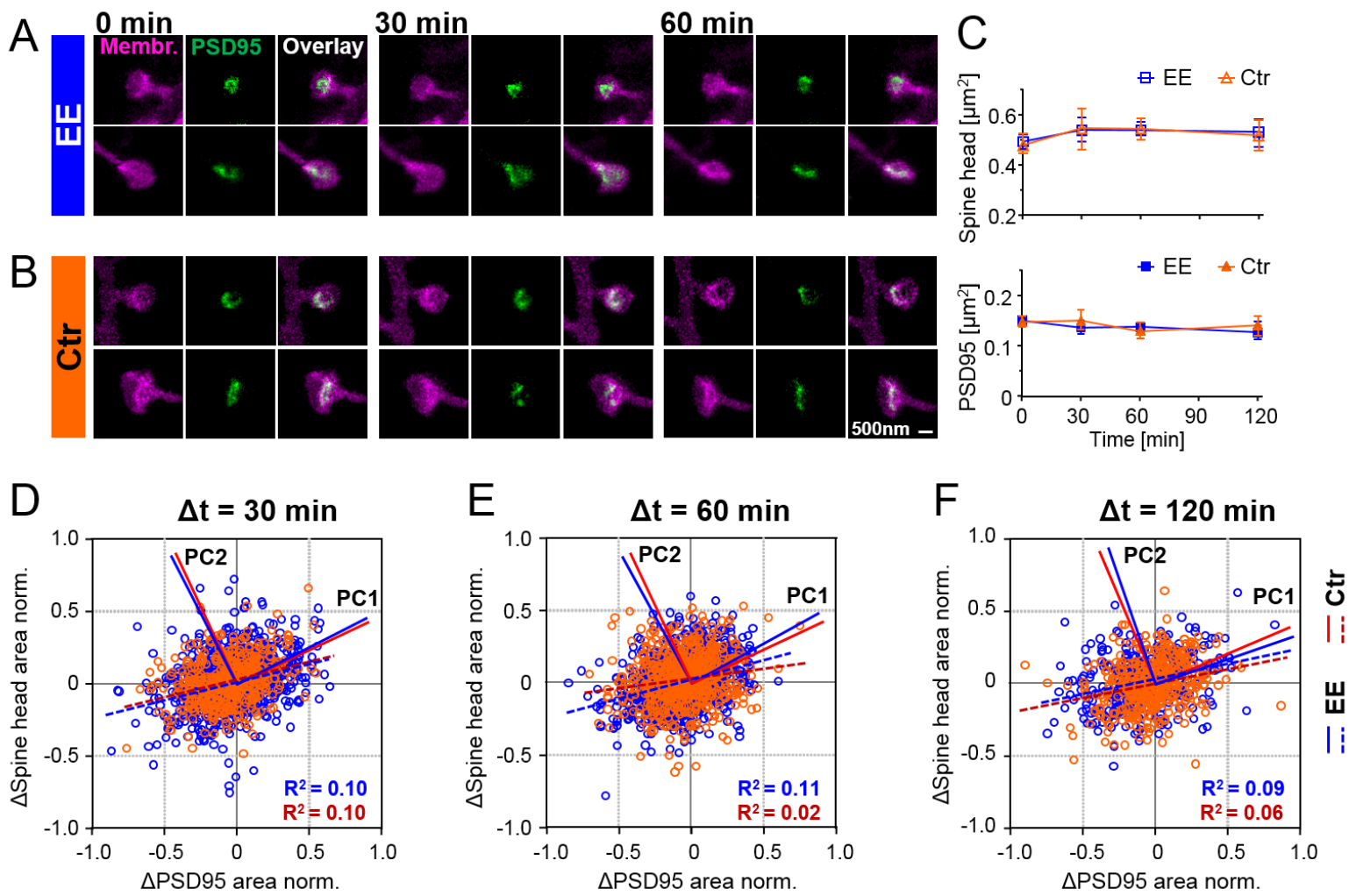

G Positive correlation

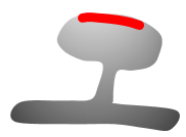

Large PSD95

Large spine

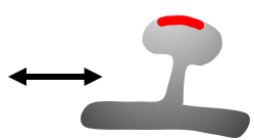

Small PSD95 Small spine
$\mathrm{H}$ Negative correlation

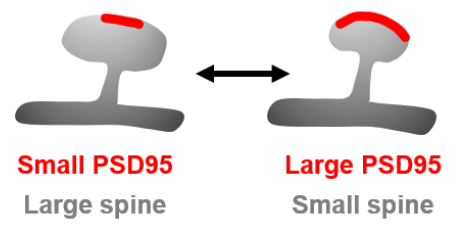

Figure 3: Temporal changes of spine head and PSD95 area are positively and negatively correlated. (A, B) Representative sections of spine heads and corresponding PSD95 of time-lapse in vivo two-color STED microscopy images. EE (A) and Ctr (B) housed mice at time points of $0 \mathrm{~min}, 30 \mathrm{~min}$ and $60 \mathrm{~min}$. Images are smoothed and shown in MIP. Scale bar $500 \mathrm{~nm}$. (C) Average of spine head areas and PSD95 areas of $\mathrm{Ctr}$ (triangle, orange) and EE (square, blue) housed mice over time. Plots show median $\pm 95 \%$ confidence interval (CI). (D-F) Normalized changes in spine head and PSD95 area after $30 \mathrm{~min}, 60 \mathrm{~min}$ and 120 min time intervals. Linear regression lines are dotted (deviation from zero: $p<0.0001$ ). Solid lines represent the principal components 1 and 2 (PC1, PC2) of the principal component analysis (PCA) for $\mathrm{Ctr}$ (red) and EE (blue), respectively. (G, H) Illustration of temporal changes between spine head size and PSD95 area. (G) Growth and shrinkage of spine head size and the area of PSD95 assemblies goes hand-in-hand, i.e. the changes are (positively) correlated. (H) An anti-correlation or negative correlation is observed for a growing PSD95 assembly on a shrinking spine and vice versa. (C-F) Number of mice and spines: $\Delta t=30 \mathrm{~min}: 4 x$ Pmice, $n_{\text {spine }}=453$ for Ctr and $4 x$ Pmice, $n_{\text {spine }}=958$ for EE; $\Delta \mathrm{t}=60 \mathrm{~min}: 4 \mathrm{x}$ \%mice, $\mathrm{n}_{\text {spine }}=646$ for Ctr and $4 \mathrm{x}$ \%mice, $\mathrm{n}_{\text {spine }}=1027$ for EE; $\Delta \mathrm{t}=120$ min: 3x 9mice, $\mathrm{n}_{\text {spine }}=386$ for Ctr and $4 \mathrm{x}$ Pmice, $\mathrm{n}_{\text {spine }}=358$ for $\mathrm{EE}$.

\section{Multiplicative downscaling of PSD95 area increases over time in EE but not Ctr housed mice}

Since our parameters were log-normally distributed, indicating a multiplicative dynamics, we asked whether the temporal changes were also regulated by multiplicative processes ${ }^{6,22}$. Thus, we plotted 
the synaptic size after different time points as a function of the original size (Fig.4A-F). A linear regression analysis revealed a slope $<1$ and positive $y$-intercept for spine head and PSD95 area, for both $\mathrm{EE}$ and $\mathrm{Ctr}$ housed mice, indicating that large spines tend to shrink and small spines tend to grow which is often denoted as multiplicative dynamics. A recent study modelling spine dynamics as a Kesten process termed the slope as shown in Fig. 4 as time-dependent multiplicative downscaling factor $^{6,41}$. Using this metric of ongoing synaptic dynamics we observed significant increase in downscaling, i.e. a decreasing slope over time for the PSD95 area of EE housed mice (Fig.4E,F) but lower downscaling over time in Ctr house mice (Fig.4B). Interestingly, this effect was specific to PSD95 as for the spine head size the slope of the linear regression did not change over time and therefore no time dependent downscaling was observed (Fig.4A,D,F). The $R^{2}$ value of the linear regression was relatively constant over all time intervals, for spine head and PSD95 area of EE and Ctr (Fig.4C). Plotting the normalized changes as a function of the normalized spine head or PSD95 area, respectively, also showed a significant change in slope over time for the PSD95 area of EE housed mice (Fig.S5A-D). In summary, we found a significant increase in downscaling over time for PSD95 size in EE mice compared to Ctr. This pronounced dynamics more strongly restores fluctuations away from the average parameters in EE in line with a more concentrated distribution of PSD95 and spine head sizes that are also associated with EE. Comparing a silenced network with an active network in vitro Hazan et al. found a significant decrease in downscaling of synaptic sizes over time after silencing and a decrease of the $\mathrm{R}^{2}$ value $^{6}$. By analogy, the more pronounced downscaling we find might be explained by a higher activity level in EE mice and potentially causing the narrower size distribution of EE housed mice (Fig.2F) ${ }^{6}$. However, we did not observe such a time-dependent downscaling for spine head size and did not observe a change in its $\mathrm{R}^{2}$ value such as described in vitro ${ }^{6}$.

\section{Faster loss of size correlation in EE housed mice}

Next, we asked how spine size change correlated over time. Do growing spines continue to grow and do shrinking spines continue to shrink? Thus, we computed the auto-correlation of the spine head and PSD95 areas over all time points (Fig.4G,H). Both the spine head areas and the PSD95 areas showed positive correlation with a large offset for both $\mathrm{EE}$ and $\mathrm{Ctr}$ housed mice. This indicates that large spine heads tend to stay rather large and smaller ones small over the observation time of up to $120 \mathrm{~min}$. However, at all time intervals the correlation was significantly smaller for EE housed mice. In other words, the spine size of EE housed mice is less similar after this time. This suggests that the spine population and attached PSD95 are able to change and adapt faster in EE housed mice. To analyze the coupling between spine head and PSD95 area changes, we computed the cross-correlation between these measures. Fig.4l shows that the cross-correlation was also lower in EE housed mice $(\Delta t=0)$ and we did not observe directional changes of these parameters. In particular, we did not observe that PSD95 increase would systematically precede a subsequent spine head increase or vice versa as both would result in an asymmetric cross-correlation. 
A Spine head area Ctr

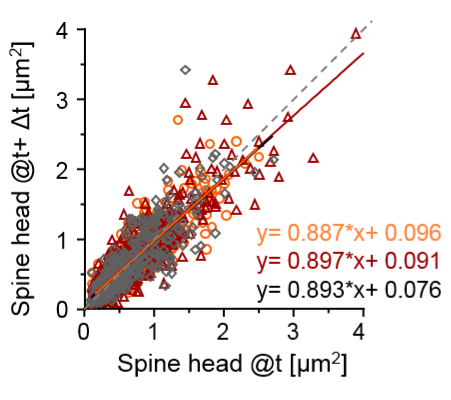

$\rightarrow \Delta \mathrm{t}=30 \mathrm{~min}$

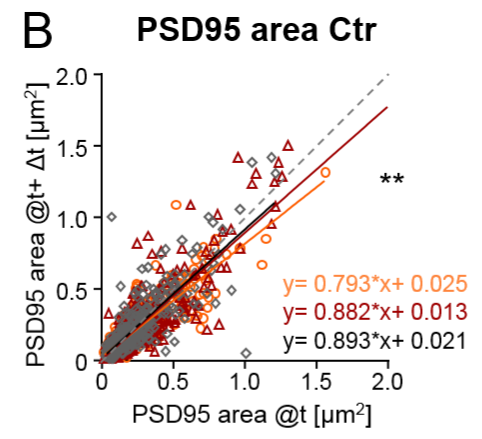

$\Delta \mathrm{t}=60 \mathrm{~min} \rightarrow \Delta \mathrm{t}=120 \mathrm{~min}$

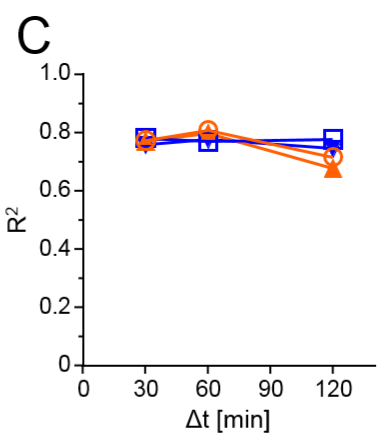

$\vartheta$ Spine head Ctr

$\boxminus$ Spine head EE

- PSD95 area Ctr

₹ PSD95 area EE
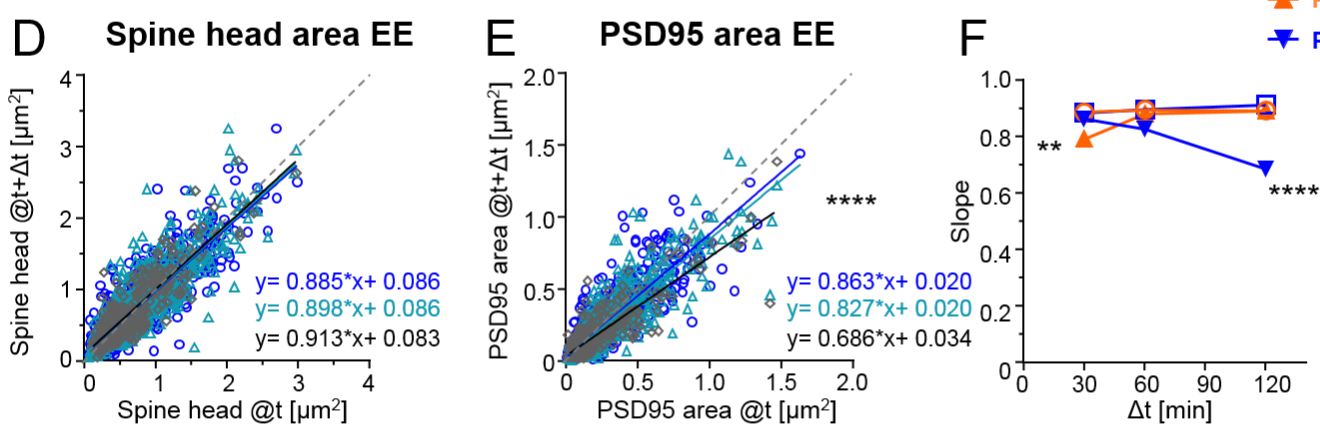

$\rightarrow \Delta t=30 \mathrm{~min}$

$\triangle \Delta \mathrm{t}=60 \mathrm{~min} \rightarrow \Delta \mathrm{t}=120 \mathrm{~min}$

G Spine head area

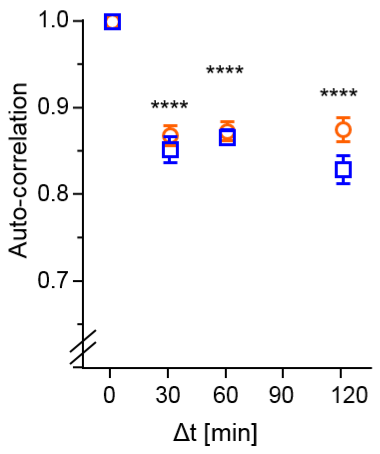

H PSD95 area

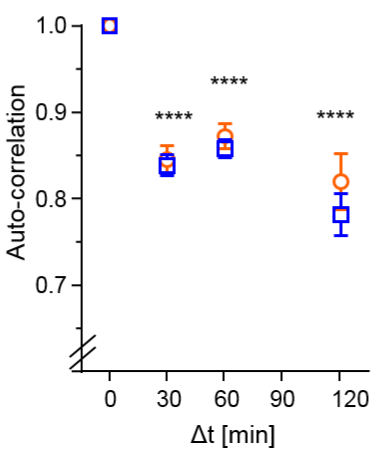

Spine head area - PSD95 area

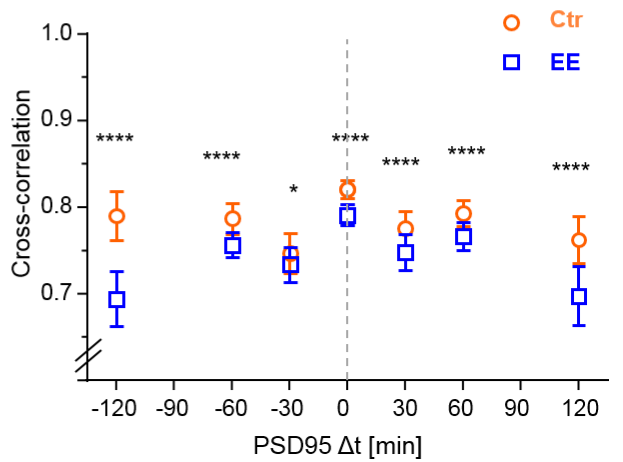

Figure 4: EE housed mice show an increase in multiplicative downscaling of PSD95 area and a lower correlation in spine head and PSD95 area over time and between these parameters. (A-F) Spine head and PSD95 area after different time intervals as function of area at $t$. Spine head area at time point $t$ and after time intervals $\Delta \mathrm{t}$ of $30 \mathrm{~min}, 60 \mathrm{~min}$ and $120 \mathrm{~min}$ for $\mathrm{Ctr}$ (A) and EE housed mice (D). Analogous plots of PSD95 area for Ctr (B) and EE housed mice (E). Solid lines shows linear regression fits of the displayed equation; the identity line is dashed $(A, B, D, E) . R^{2}$ value $(C)$ and slope $(F)$ of linear regressions $(A, B, D, E)$ for different time intervals $\Delta t$. (Analysis of Covariance, Slopes equal? $A: p=0.95, B:{ }^{* *} p=$ 0.005, D: $p=0.64, E: * * * p<0.0001)$. (G-I) Auto-correlation of spine head (G) and PSD95 area (H) and cross-correlation (I) of these parameters for different time intervals $\Delta t$ (Unpaired $t$-test with Welch's correction, $* p<0.05,{ }^{* * * *} p<0.0001$ ). Error bars are bootstrap SD. (A-I) Same data as Fig. 3.

\section{PSD95 nanoorganization changes faster in EE mice}

Previous works with EM have shown that PSDs on large spines, often called mushroom spines, are frequently perforated ${ }^{25}$. In a previous publication ${ }^{30}$, we investigated PSD95 morphology in homozygous 
knock-in mice and showed that PSD95 assemblies on large spines were also often perforated and appeared ring-like or clustered. This nanoorganization was highly dynamic and changed in shape within a few hours. Now, we asked whether these changes would be different for mice housed in EE. Therefore, we selected perforated PSD95 assemblies and investigated their morphological changes analogously to the study by Wegner et al. ${ }^{30}$. Fig.5A shows examples of two-color STED images of perforated PSD95 assemblies and their associated spine revealing temporal changes in the PSD95 nanoorganization. Morphological changes were categorized as no changes, subtle changes, or strong changes. The classification of morphological changes was performed blinded by three independent persons and the changes were analyzed with respect to their morphology at baseline $t=0$ (Fig.5B,C). Ctr mice showed a clear trend: the longer the measurement interval, the greater the morphological changes (Fig.5C). Within the time interval of 30-120min, the percentage of PSD95 nanoorganization showing no change decreased from $\sim 40 \%$ to $\sim 10 \%$, while the percentage for strong changes nearly tripled for Ctr. For EE housed mice, we observed similar changes within all investigated time intervals; $\sim 20 \%$ of the PSD95 assemblies did not change at all and $\sim 80 \%$ underwent a change at all time points (Fig.5B). Although the percentage changes with respect to $\mathrm{t}=0$ were similar for different time intervals, it should be noted that this does not exclude dynamic changes between the time points. Since we observe an equilibrium of changes in $\mathrm{EE}$ mice but not in Ctr mice, it argues for a time scale of less than $30 \mathrm{~min}$, i.e. our shortest observation interval, to perform a change in EE mice but approximately $30 \mathrm{~min}$ in Ctr mice. These results show that not only the PSD95 total size but also the PSD95 nanoorganization is more dynamic in EE mice than in mice raised under control conditions within the investigated time course. Now, we asked whether this dynamic would be reflected as well in a different nanoorganization. Therefore, the nanoorganization was analyzed at $\mathrm{t}=0$ and categorized into perforated (a continuous shape with a sub-structure such as ring- or horseshoe-like) or clustered. Indeed, fewer nanoorganizations of EE housed mice were perforated and a larger fraction was showing three or more clusters compared to Ctr mice (Fig.5D). This suggest that the PSD95 nanoorganization might play a substantial role in synaptic remodelling and is specifically shaped by experience or activity. 


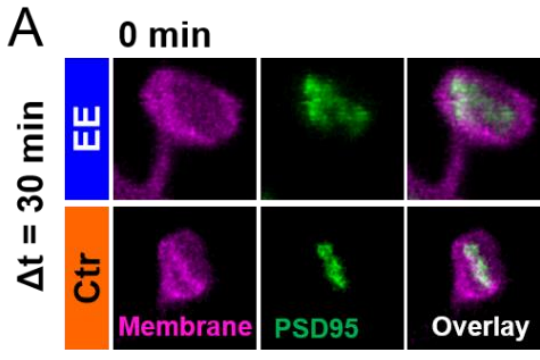

$30 \mathrm{~min}$
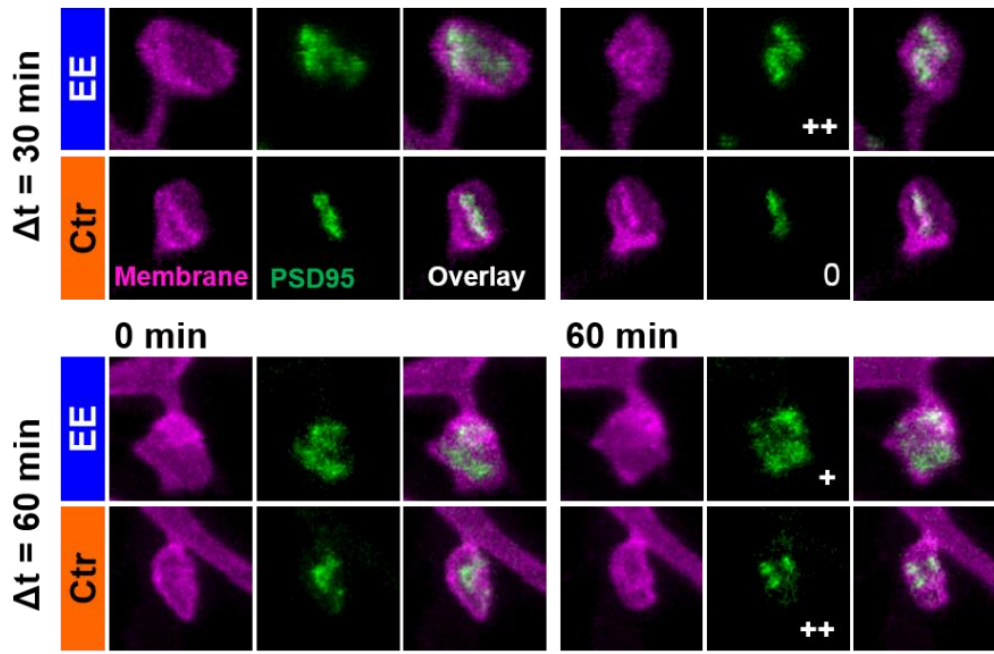

$60 \mathrm{~min}$
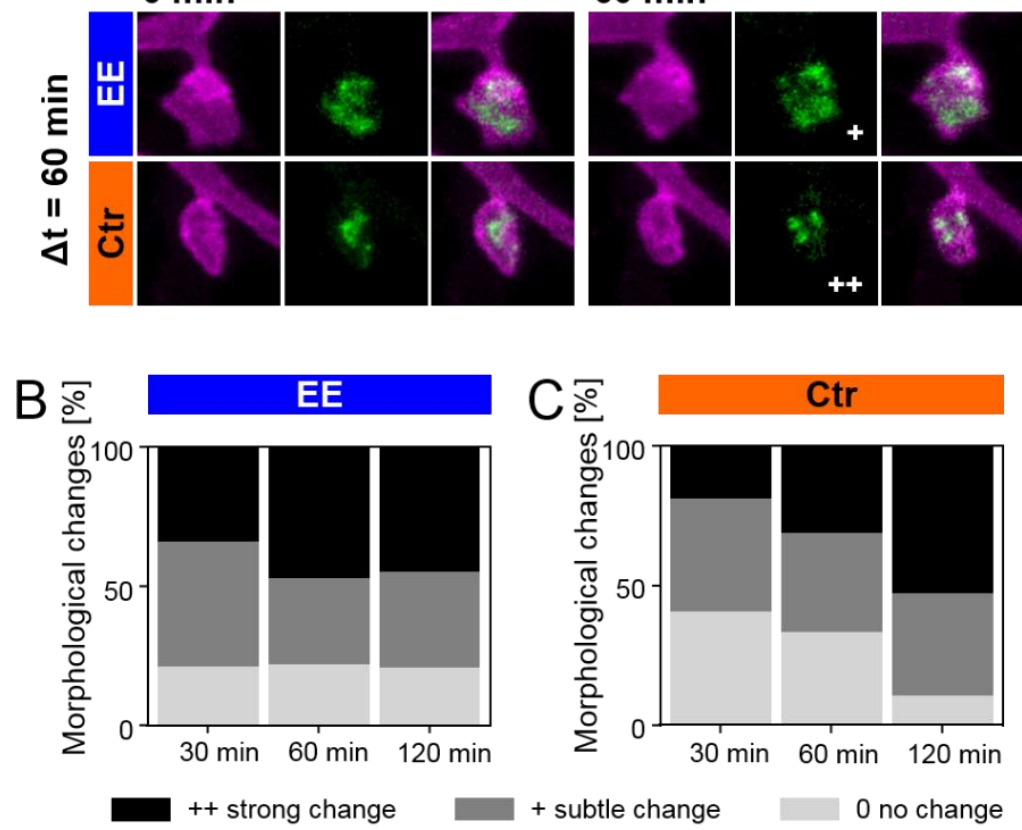

$60 \min$

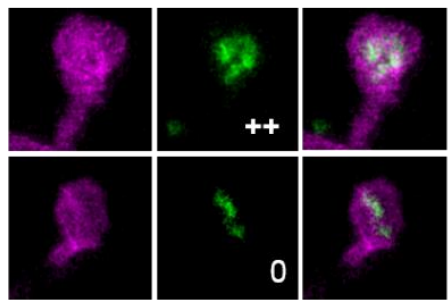

$120 \mathrm{~min}$

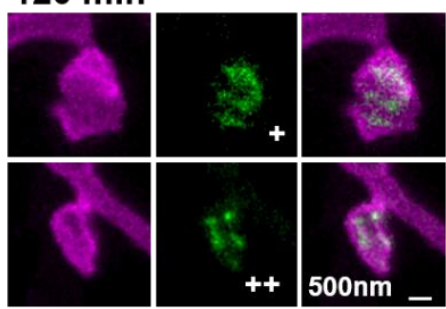

Figure 5: PSD95 nanoorganization is different between EE and Ctr and changes faster for EE housed mice. (A) Sections of two-color STED images (smoothed, MIP) of EE and Ctr housed mice at the indicated time points showing the spine membrane (magenta) and PSD95 (green). Morphological change is indicated with: $\mathbf{0}=$ no change; $+=$ subtle change; $++=$ strong change. (B, C) Stacked histogram of the relative frequency of morphological changes in PSD95 nanoorganization in EE (B) and Ctr (C) housed mice; all changes refer to $\mathrm{t}=0 \mathrm{~min}$. (D) Morphometry of PSD95 nanoorganization of EE and $\mathrm{Ctr}$ housed mice at $\mathrm{t}=0$. Number of analyzed PSD95 assemblies in EE: $30 \mathrm{~min}: \mathrm{n}=38,60 \mathrm{~min}: \mathrm{n}=68,120$ $\min : n=29 ;$ Ctr: $30 \min : n=27,60 \min : n=48,120 \min : n=38$.

\section{DISCUSSION}

Interactions with the environment play a key role in restructuring and refining the neuronal circuitry in the brain. Previous work has shown that an enriched environment results in structural as well as functional changes of synapses ${ }^{2,18,20,42}$ and stimulates synaptic plasticity in the mouse visual cortex ${ }^{33}$. However, most studies were performed post mortem or ex vivo in slices. For the first time we here used in vivo STED microscopy, a superresolution light microscopy technique with nanoscale resolution, to perform an analysis of synaptic morphology as well as its nanoplasticity in the intact brain of EE housed and control mice. We designed a two-color, virtually crosstalk free in vivo STED microscope with a resolution of 70-80nm for simultaneous imaging of spine morphology and PSD95 in the visual cortex of anesthetized mice. We found a significantly smaller variance in size of the spine head and of 
the post-synaptic density in EE mice while their average sizes were similar to Ctr mice. Both parameters were highly volatile and we observed a growth of $>23 \%$ and shrinkage of $>17 \%$ for spine heads and PSD95 assemblies within $30 \mathrm{~min}$. About $2 / 3$ of these changes were positively correlated and $\sim 1 / 3$ showed a negative correlation; the amplitude of changes did not differ clearly between $\mathrm{EE}$ and Ctr. All parameters exhibited multiplicative downscaling, which is an indication for multiplicative changes. PSD95 was more dynamic in EE mice as denoted by an increase in multiplicative downscaling over time, faster decrease in size correlation over time and faster changes of the nanoorganization.

\section{Two-color STED nanoscopy of synaptic nanoorganization}

Previous studies mainly used two-photon microscopy to study synapse and spine plasticity in vivo and in vitro ${ }^{13,14,16,43}$. Due to its limited resolution of $300-500 \mathrm{~nm}$, spine brightness is often taken as a measure of size $e^{12,16,23}$. This is not very accurate since the brightness can be influenced by many factors such as expression level of the fluorescence label, the excitation laser intensity, group velocity dispersion or scattering in the tissue. Therefore, spine brightness is typically normalized to the brightness of the dendrite, making comparative studies and determination of absolute sizes difficult. Two-photon microscopy can be combined with STED to achieve nanoscale resolution ${ }^{44,45}$. However, a drawback of this approach is the relatively high crosstalk when using two colors. The excitation and emission of the fluorescent labels must be spectrally close to each other in order to use the same twophoton excitation and stimulated emission laser for both fluorophores ${ }^{45}$. Thus for EGFP and EYFP that are typically used, the reported crosstalk is as high as $92 \%$ and $27 \%$, respectively, requiring linear unmixing ${ }^{21}$. Unmixing works well for structures which are relatively bright and spatially separated such as for volume labeled spines and astrocytes ${ }^{45}$ but can produce artefacts in dark and overlapping structures. Here, we performed STED microscopy of an endogenously tagged synaptic protein, which has a considerably lower brightness than a volume label and spatially overlaps with the second label, the spine membrane, and therefore unmixing would be difficult. We achieved this by designing a twocolor STED microscope with two different excitation lasers (one-photon excitation) and two spectrally separated detection channels. This new approach gave us an almost negligible crosstalk of $5 \%$ for EGFP and $8 \%$ for Citrine and making unmixing obsolete.

\section{Temporal changes of PSD95 assemblies and spine heads are largely uncorrelated}

It has been shown that PSD95 overexpression promotes the maturation of glutamatergic synapses, increases spine size, and enhances AMPA receptor currents ${ }^{46,47}$. Therefore, we used the transcriptionally regulated antibody-like PSD95-FingR, which efficiently labels endogenous PSD95 ${ }^{31,48}$ and excludes overexpression artefacts. While we observed a substantial correlation between the absolute spine head and PSD95 assembly size ( $\mathrm{R}^{2 \sim 0.7}$, Fig.2B), corroborating previous EM studies ${ }^{15,17}$, we found only weak correlations between their temporal changes $\left(R^{2} 0.1\right.$, Fig.3D-F). Most of the changes were positively correlated while about $\sim 1 / 3$ was attributed to negatively correlated changes. LTP experiments have shown a rapid increase in spine head size and AMPA receptor current but a delayed increase of PSD95 and other scaffolding proteins of $\sim 60 \mathrm{~min}^{13,14}$. Although these experiments were performed in cell culture and with overexpressions, they indicate that spine and PSD changes might not be temporally concerted and therefore might be regulated differently ${ }^{10,11}$. These results are thus congruent with the largely uncorrelated changes we observed at baseline in vivo at similar time scales of $30-120 \mathrm{~min}$. However, we also found no directional changes that would demonstrate the PSD95 size to systematically follow an increase in spine head size, as the cross-correlation is symmetric (Fig.4I). Nevertheless, why is the time-line of AMPA receptor incorporation and PSD95 increase so different although AMPA receptors are linked to PSD95 by TARPs? At the moment different hypotheses are discussed. There is evidence that LTP promotes the exocytosis of AMPA receptor containing vesicles and thus increases the number of receptors at the synapse ${ }^{10}$. On the other hand, a slot model 
proposes that the actin polymerization during LTP increases the number of slots which can harbor AMPA receptors ${ }^{10}$. Both hypotheses are in line with our observations. However, we found that spine heads and PSD95 area changed by about the same percentage of $>23 \%$ already after $30 \mathrm{~min}$. Meyer et al. reported a three times larger increase in spine volume than PSD95 volume after LTP and only 19\% of change in PSD95 volume after one hour ${ }^{14}$. While the overexpression of PSD95 might have influenced the results by Meyer et al. we observe a PSD95 baseline volatility which is in the range of LTP driven changes.

\section{Synapses of EE housed mice are sharper defined in size and PSD95 assemblies change more rapidly}

We next compared adult mice housed in standard cages after the critical period with age matched EE housed mice and analyzed synaptic morphology and plasticity. All histograms of spine head and PSD95 area were positively skewed and log-normally distributed. While the sizes were similar between EE and Ctr housed mice, their variances were different. Both distributions, spine head and PSD95 area, were significantly narrower or sharper in EE housed mice. At the moment different models of how neuronal networks adapt to changes in activity to undergo homeostatic plasticity are considered ${ }^{49}$. In the model of synaptic scaling, an increase in activity leads to a downscaling of excitatory synapses. The downscaling is multiplicative and therefore changes affect the average as well as broadening of the size distribution as shown, for example, in a silenced network ${ }^{6}$. In vivo and with $\mathrm{EE}$, however, we observed no change in average size and therefore not pure synaptic scaling. This is in line with recent in vivo observations that in the adult cortex homeostatic plasticity is rather input-specific and not multiplicative for the whole ensemble of synapses ${ }^{49}$.

Changes of spine heads and PSD95 assemblies correlated with their absolute size for EE and Ctr house mice. Small spines tended to grow and large spines tended to shrink. Such morphological changes can be modeled by a Kesten process including additive and multiplicative changes. This model entails a time-dependent multiplicative downscaling factor ${ }^{6}$ corresponding to the slope in Fig.4A-D. Silencing a neuronal network not only resulted in an average size increase and broader distribution but also in a weaker multiplicative downscaling ${ }^{6}$. Our observations are in line with these results in such that the increase in activity by enrichment strengthened the multiplicative downscaling over time for PSD95. This in turn explains the narrower size distribution. However, we did not observe these changes between $\mathrm{EE}$ and $\mathrm{Ctr}$ for the spine head. We note in passing that it should not be surprising that our in vivo experiments do not completely match expectations from artificially silenced neuronal culture experiment. As discussed above, there is evidence that homeostatic plasticity is not global but inputspecific $^{49}$.

\section{Enhanced nanoplasticity by experience}

With STED nanoscopy we often observed a perforated nanoorganization of PSD95 undetectable by two-photon imaging. In EE housed mice, the nanoorganization of PSD95 was different and more dynamic. Accumulating evidence indicate that pre- and postsynaptic structures are clustered. The functional consequence of this clustering, however, are not fully understood. An emerging view is that these clusters are trans-synaptically aligned in 'nanocolumns' which are organized by activity ${ }^{50}$. Interestingly, this implies that a reorganization of receptors might alter synaptic strength independently of changes in the amount of receptors ${ }^{50}$. Our finding of an increased structural plasticity of the PSD95 nanoorganization but not increased average size in EE mice might therefore reflect an increase of synaptic plasticity ${ }^{42,51}$.

\section{Outlook}

Our results demonstrate that two-color STED microscopy opens novel avenues for the in vivo investigation of the synaptic nanoorganization and its dynamics in the living brain. Our two-color STED 
approach can be extended in the future to study the dynamics of further synaptic supramolecular complexes and uncover the plasticity of nanocolumns after experience, as well as over longer timeintervals in chronic experiments and different brain regions. The crosstalk free two-color imaging introduced here will be critical to obtain unequivocal observations on the dynamics of synaptic molecular architecture in the living brain.

\section{MATERIALS AND METHODS}

\section{DNA constructs}

The transcriptionally regulated antibody-like protein to label PSD95 was obtained via plasmid pAAVZFN-hSyn-DIO-PSD95-FingR-Citrine-CCR5TC, with a double-floxed inverted open reading frame (DIO). It was cloned sequentially by creating the following plasmids. 1) pAAV-hSyn-DIO-EYFP: PCR based insertion of the human Synapsin promoter (hSyn) instead of the human elongation factor-1 alpha (Ef1a) promoter into the vector backbone of pAAV-Ef1a-DIO-EYFP, kindly provided by the laboratory of Dr. K. Deisseroth of Stanford University, CA. 2) pAAV-ZFN-hSyn-DIO-EYFP: Insertion of the zinc finger binding motif (ZFN) ${ }^{31} 5^{\prime}$ to the hSyn promoter by hybridization of two $5^{\prime}$ phosphorylated primers. 3) pAAV-ZFN-hSyn-DIO-PSD95-FingR-Citrine-CCR5TC: PCR based insertion of the antibody like PSD95FingR (FingR: Fibronectin intrabodies generated with mRNA display) ${ }^{31}$, the transcriptionally regulatory elements CCR5ZFL-KRAB(A) (CCR5ZFL: left zinc finger from an engineered zinc-finger nuclease against the gene $C C R 5 ; \operatorname{KRAB}(A)$ : transcriptional repressor domain from rat Kid-1 ${ }^{31}$ ), and Citrine to obtain the final plasmid. The sequences of PSD95-FingR and CCR5ZFL-KRAB(A) were amplified from pCAG_PSD95.FingR-eGFP-CCR5TC (gift from Dr. Don Arnold (Addgene plasmid \# 46295)). All primers and restriction endonucleases used for cloning are listed in Table 1.

The plasmid pAAV-hSyn-DIO-myr-EGFP-LDLR(Ct) for dendritic membrane labelling was cloned as follows: first, we PCR amplified a myristoylation (myr) site-attached EGFP including the myristoylation sequence ATGGGCTGTGTGCAATGTAAGGATAAAGAAGCAACAAAACTGACG in the forward primer. Second, we split the C-terminal (Ct) cytoplasmic domains of low density lipoprotein receptor (LDLR; gb: AF425607, amino acid residues 813-862) ${ }^{38}$, finally designated as LDLR(Ct), into two parts (part 1 and part 2), designed $5^{\prime}$ phosphorylated forward and reverse primers for each part and hybridized each pair (Table 1). In the third and final step, the endonuclease-digested myr-EGFP PCR together with the LDLR(Ct) part1 and LDLR(Ct) part 2 were ligated into plasmid pAAV-hSyn-DIO-EYFP digested with Ascl and Nhel to finally obtain pAAV-hSyn-DIO-myrEGFP-LDLR(Ct). Generation of the Cre recombinase expression plasmid pAAV-hSyn-Cre was performed as previously described ${ }^{52}$. To determine the crosstalk of our two-color in vivo STED microscope, we used two plasmids, both encoding a fusion protein consisting of the small peptide Lifeact (LA), that directly binds to F-actin, and a fluorescent protein: pAAV-hSyn-LA-EYFP ${ }^{34}$ and pAAV-hSyn-LA-EGFP. pAAV-hSyn-LA-EGFP was generated by the exchange of the fluorescent protein EYFP with EGFP. All generated or used AAVs contain as the 3' untranslated regions the woodchuck hepatitis virus posttranslational regulatory element (WPRE) and the human growth hormone polyadenylation site.

Table 1: Overview of the primers and endonucleases used to clone new plasmids. a: produced by PCR, b: generated by hybridization, $\mathrm{P}$ : phosphorylated; underlined nucleotides: restriction sites or part of them 


\begin{tabular}{|c|c|c|c|}
\hline $\begin{array}{l}\text { Name of the plasmid to } \\
\text { be generated }\end{array}$ & Primers & $\begin{array}{l}\text { Restriction } \\
\text { sites }\end{array}$ & DNA-insert \\
\hline \multirow{2}{*}{ pAAV-hSyn-DIO-EYFP } & $5^{\prime}-$ ccgtacgcgtgtgtctagactgcagagggecctg $-3^{\prime}$ & MluI & \multirow{2}{*}{$\mathrm{hSyn}^{\mathrm{a}}$} \\
\hline & $5^{\prime}$ - gcatgtcgacggatccggtaccttctcgactgcgc $-3^{\prime}$ & SalI & \\
\hline \multirow{2}{*}{$\begin{array}{l}\text { pAAV-ZFN-hSyn-DIO- } \\
\text { EYFP }\end{array}$} & P-5'-cgcgtgtcatcctcatcgtgt $-3^{\prime}$ & $\mathrm{MluI}+\mathrm{XbaI}$ & \multirow{2}{*}{$\mathrm{ZFN}^{\mathrm{b}}$} \\
\hline & P-5'-ctagacacgatgaggatgaca $-3^{\prime}$ & MluI + XbaI & \\
\hline \multirow{6}{*}{$\begin{array}{l}\text { pAAV-ZFN-hSyn-DIO- } \\
\text { PSD95-FingR-Citrine- } \\
\text { CCR5TC }\end{array}$} & $5^{\prime}$ - gacaccatggatctactggagccgctacc $-3^{\prime}$ & NcoI & \multirow{2}{*}{ PSD95-FingR $^{\mathrm{a}}$} \\
\hline & $5^{\prime}$ - tccgtgctagcatgctcgaagtcaaggaagc $-3^{\prime}$ & NheI & \\
\hline & $5^{\prime}-$ atcggcgcgecttaagccatagaagcaagattag $-3^{\prime}$ & AscI & \multirow{2}{*}{$\begin{array}{l}\text { CCR5ZFL- } \\
{\mathrm{KRAB}(\mathrm{A})^{\mathrm{a}}}^{\mathrm{a}}\end{array}$} \\
\hline & $5^{\prime}$ - gctggcgccggagcgggtgcaggggc $-3^{\prime}$ & KasI & \\
\hline & $5^{\prime}$ - agcggcgccagctcccctagacttgtacagctcgtc $-3^{\prime}$ & KasI & \multirow{2}{*}{ Citrine $^{\mathrm{a}}$} \\
\hline & $5^{\prime}$ - gatccatggtgagcaagggcgaggagctgttcac $-3^{\prime}$ & NcoI & \\
\hline \multirow{2}{*}{ pAAV-hSyn-LA-EGFP } & $5^{\prime}$ - gatccatggtgagcaagggcgaggagctgttcac $-3^{\prime}$ & NcoI & \multirow{2}{*}{ EGFPa $^{a}$} \\
\hline & $5^{\prime}$ - gcgccgaattcttacttgtacagctcgtccatgc $-3^{\prime}$ & EcoRI & \\
\hline \multirow{6}{*}{$\begin{array}{l}\text { pAAV-hSyn-DIO- } \\
\text { myrEGFP-LDLR(Ct) }\end{array}$} & $\begin{array}{l}5^{\prime} \text { - agttatgctagcatgggctgtgtgcaatgtaaggataaag } \\
\text { aagcaacaaaactgacgatggtgagcaagggcgaggag }-3^{\prime}\end{array}$ & NheI & \multirow[t]{2}{*}{$\begin{array}{l}\text { Myristol (myr) } \\
\text { EGFPa }^{a}\end{array}$} \\
\hline & $5^{\prime}$ - cgcaccggtcttgtacagctcgtccatg-3' & AgeI & \\
\hline & $\begin{array}{l}\text { P-5' -ccggtcggaactggegectgaagaatatcaacagc } \\
\text { atcaatttcgataaccccgtgtaccagaagaccacagaggat }-3^{\prime}\end{array}$ & AgeI & $\operatorname{LDLR}(\mathrm{Ct})-$ part $^{\mathrm{b}}$ \\
\hline & $\begin{array}{l}\text { P-5' -cagctcatcctctgtggtcttctggtacacggggttatcgaaa } \\
\text { ttgatgctgttgatattcttcaggcgecagttccga }-3^{\prime}\end{array}$ & AgeI & $\operatorname{LDLR}(\mathrm{Ct})-$ part $1^{\mathrm{b}}$ \\
\hline & $\begin{array}{l}\text { P-5' -gagctgcacatttgcaggtcccaagacgggtacacctatcc } \\
\text { aagtcggcagatggtcagcctcgaggacgatgtggectgagg }-3 \text { ' }\end{array}$ & AscI & $\operatorname{LDLR}(\mathrm{Ct})-$ part $2^{\mathrm{b}}$ \\
\hline & $\begin{array}{l}\text { P-5' - cgcgectcaggccacatcgtcetcgaggctgaccatctgcc } \\
\text { gacttggataggtgtaccegtcttgggacctgcaaatgtg }-3^{\prime}\end{array}$ & AscI & $\operatorname{LDLR}(\mathrm{Ct})-$ part2 ${ }^{\mathrm{b}}$ \\
\hline
\end{tabular}

\section{Virus production}

Recombinant rAAV particles with mixed serotype 1 and 2 of pAAV plasmids encoding the proteins of interest were produced in HEK293-FT cells. The entire procedure was previously described in detail ${ }^{52}$ and is applied here with the following modifications: After DNasel treatment ( 30 min at $37^{\circ} \mathrm{C}$ ), the suspension was centrifuged at $1200 \mathrm{~g}$ for $10 \mathrm{~min}$. The supernatant was filtered through an $0.45 \mu \mathrm{m}$ sterile filter (Merck/Millipore, Darmstadt, Germany) and first applied to an Amicon Ultra-15, MWCO $100 \mathrm{kDa}$, centrifugal filter unit (Merck/Millipore, Darmstadt, Germany), followed by a Vivaspin ${ }^{\circledR} 500$, MWCO $100 \mathrm{kDa}$, centrifugal concentrator (Sartorius, Göttingen, Germany) to wash the virus in Opti-

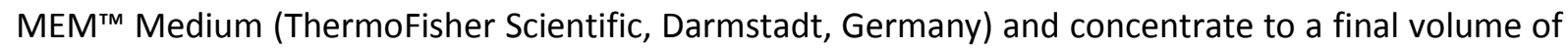
$150 \mu \mathrm{l}$.

\section{Animals}

All animal experiments were performed with C57BL/6J female mice reared at the animal facility of the Max Planck Institute of Experimental Medicine in Göttingen and housed with a 12-h light/dark cycle, with food and water available ad libitum. Experiments were performed according to the guidelines of the national law regarding animal protection procedures and were approved by the responsible 
authorities, the Niedersächsisches Landesamt für Verbraucherschutz (LAVES, identification number 33.9-42502-04-14/1463). All efforts were made to minimize animal suffering and the number of animals used.

\section{Housing conditions}

Environmentally enriched (EE) animals were born and raised in the commercially available Marlau ${ }^{\mathrm{TM}}$ cage (Marlau ${ }^{\mathrm{TM}}$, Viewpoint, Lyon, France) ${ }^{53}, 580 \times 400 \times 320 \mathrm{~mm}$ in size including 2 floors, providing an extensive exploration area (Fig. S2). Three pregnant female mice were placed in an EE cage about 1 week before delivery. Pups were weaned and split by sex at post-natal day 30 . The ground floor of the EE cage consisted of two separate compartments: the smaller part contained food and the larger part contained water, running wheels and a red house. To get food, mice had to use a stairway to reach the second floor where a maze was placed. After passing the maze, mice slided through a tunnel back to the ground floor, directly into the smaller compartment and had free access to food. By passing a oneway door they could enter the larger part of the ground floor to get water. To increase novelty and maintaining cognitive stimulation, the maze was changed 3 times per week with a total of 12 different configurations. Control mice (Ctr) were born and raised in standard cages of $365 \times 207 \times 140 \mathrm{~mm}$ in size. They were kept in 2-3 animals per cage, which were solely equipped with nesting material.

\section{AAV transduction}

Adult (>12 weeks) female C57BL/6J mice were stereotaxically transduced with a mixture of $\mathrm{rAAV}$ with mixed serotype 1 and 2. rAAV1/2-ZFN-hSyn-DIO-PSD95-FingR-Citrine-CCR5TC, rAAV1/2-hSyn-Cre and rAAV1/2-hSyn-DIO-myrEGFP-LDLR(Ct) were stereotaxically injected at the same time into the visual cortex of the left hemisphere at a depth of $\sim 500 \mu \mathrm{m}$ below the pia transducing mainly layer 5 pyramidal neurons by using the following coordinates: $3.4 \mathrm{~mm}$ posterior to the bregma, $2.2 \mathrm{~mm}$ lateral to the midline, at an angle of $70^{\circ}$ to the vertical, and with a feed forward of $\sim 750 \mu \mathrm{m}$. Anesthesia was initiated by intraperitoneal injection of $0.05 \mathrm{mg} / \mathrm{kg}$ fentanyl, $5 \mathrm{mg} / \mathrm{kg}$ midazolam, and $0.5 \mathrm{mg} / \mathrm{kg}$ medetomidin (MMF). The mouse was head fixed with a stereotaxic frame and placed on a heating pad throughout the whole procedure to maintain a constant body temperature. The depth of the anesthesia was controlled by monitoring the pulse rate and $\mathrm{O}_{2}$ saturation with a pulse oximeter at the thigh of the mouse and body temperature with a rectal temperature probe. A gas mixture with a high $\mathrm{O}_{2}$ (47.5 vol\%) and $\mathrm{CO}_{2}$ (2.5 vol\%) content was applied to the mouse's nose, significantly increasing the oxygenation. The eyes of the mouse were covered with eye ointment and the head was disinfected with $70 \%$ ethanol. The skin was cut by a $\sim 0.5 \mathrm{~cm}$ long incision and a drop of local anesthetic $(0.2 \mathrm{mg}$ mepivacaine) was applied. Then, a $0.5 \mathrm{~mm}$ hole was drilled into the skull. $150 \mathrm{nl}$ of rAAV containing solution was pressure injected through a glass micropipette attached to a microinjector (Picospritzer ${ }^{\circledast}$ III, Parker Hannifin Corp, Cleveland, Ohio) with an injection rate of $\sim 50 \mathrm{nl}$ per minute. After injection, the pipette was kept at the target location for an additional 2 minutes to allow the virus to disperse away. After retracting the micropipette, the incision was closed with a suture. Anesthesia was then antagonized by intraperitoneal administration of $0.1 \mathrm{mg} / \mathrm{kg}$ buprenorphine and $2.5 \mathrm{mg} / \mathrm{kg}$ atipamezole. The mouse was kept in a separate cage until full recovery and then put back into its original cage, and group-housed in the animal facility until the final experiment.

\section{Craniotomy for in vivo STED microscopy}

Three to six weeks after viral transduction, a craniotomy was performed as described previously ${ }^{39}$. In brief, the mouse was anaesthetized with MMF, placed on a heating pad and vital functions and depth of anesthesia were controlled throughout the final experiment as described above. The head was mounted in a stereotaxic frame. The scalp was removed and a flat head bar was glued to the right hemisphere to leave enough space for the cranial window above the visual cortex, rostral to the former 
viral injection site. After drilling a circular groove $(\sim 2-3 \mathrm{~mm})$ into the skull, the bony plate was carefully removed without causing a trauma. The remaining dura and arachnoid mater were carefully removed with a fine forceps. A small tube was positioned at the edge of the opening to drain excess cerebrospinal fluid if necessary. The craniotomy was sealed with a $6 \mathrm{~mm}$ diameter cover glass glued to the skull. The mouse was mounted on a tiltable plate which can be aligned perpendicular to the optical axis of the microscope in a quick and easy routine before being placed under the microscope ${ }^{39}$.

\section{Culture of primary hippocampal neurons, transduction and imaging}

Primary cultures of rat hippocampal neurons were prepared from PO-P1 Wistar rats (RjHan:WI; The Jackson Laboratory, Bar Harbor, ME) of both sexes according to ${ }^{54}$. Neurons were cultured at $37{ }^{\circ} \mathrm{C}$ in a humidified atmosphere with $5 \% \mathrm{CO}_{2}$ and transduced between 8 and 10 days in vitro (DIV) with the respective rAAVs. After an incubation time of 7 days, live cell STED imaging was performed at room temperature.

\section{In vivo two-color STED microscope}

A previously described custom-designed STED microscope ${ }^{30,34}$ was modified to accommodate for virtually crosstalk free two-color imaging as follows (Fig. 1). Excitation light (Exc1) provided by a pulsed laser diode emitting blue light at $483 \mathrm{~nm}$ (PiLas, Advanced Laser Diode Systems, Berlin, Germany) was complemented by a second excitation beam (Exc2). The beam of a Ti:Sapphire laser (MaiTai; SpectraPhysics, Santa Clara, CA) was split into two, pumping an optical parametric oscillator (OPO; APE, Berlin, Germany) emitting $80 \mathrm{MHz}$ pulses at $595 \mathrm{~nm}$ for stimulated emission depletion (STED) and a supercontinuum device (FemtoWHITE800, NKT photonics, Birkerød, Denmark) generating white light. The white light was spectrally filtered for green light with a bandpass filter (BP1, BrightLine HC 520/5, Semrock, IDEX Health \& Science, Rochester, NY) for selective excitation of EYFP or Citrine at $520 \mathrm{~nm}$ (Exc2, Fig. 1A,B). Exc1, Exc2 and the STED beam were co-aligned with dichroic mirrors. After passing a Yanus scan head (Till Photonics-FEl, Gräfelfing, Germany) consisting of two galvanometric mirrors for $x-/ y$-scanning and relay optics, the three beams were focused by a 1.3 numerical aperture objective lens (PL APO, 63x, glycerol; Leica, Wetzlar, Germany). Additionally, the STED beam was passing a vortex phase plate (VPP; RPC Photonics, Rochester, NY) to create a doughnut shaped focal intensity pattern featuring zero intensity in the center. Temporal overlap of all three pulsed laser beams was achieved electronically by synchronizing the blue laser diode, Exc1, to the MaiTai and optically by an optical delay line for Exc2. Z-scanning was performed by moving the objective with a piezo (MIPOS 100PL; piezosystem jena $\mathrm{GmbH}$, Jena, Germany). The back-projected fluorescence light was split at $515 \mathrm{~nm}$ with a dichroic mirror (DM2, ZT502rdc-UF3; Chroma Technology Corporation, Bellow Falls, VT) into two beams. The shorter wavelength was reflected, filtered by a bandpass filter (BP3, BrightLine HC 504/12; Semrock) and focused onto a multimode fibre for confocal detection connected to an avalanche photodiode (APD, Excelitas, Waltham, MA). The transmitted, longer wavelength fluorescence light was also filtered with a bandpass filter (BP2, H544/23, AHF analysentechnik, Tübingen, Germany) and detected with an APD, respectively.

\section{In vivo two-color STED imaging of anesthetized mice}

The in vivo STED microscopy was performed in layer 1 of the visual cortex at a depth of 5-20 $\mu \mathrm{m}$ below the pia. Spherical aberrations due to the tissue penetration were corrected on the first order by adapting the correction collar of the glycerol immersion objective for the best image quality at each field of view. Both excitation colors were alternated line-by-line; i.e. a line was recorded by excitation with blue light (Exc1) and then the same line was recorded again with green excitation light (Exc2). Potential drift between images and movement of the spine or PSD95 was therefore negligible. For imaging we picked FOVs with dendrites parallel to the focal plane. After recording a two-color STED 
image stack, the position of the motorized micrometer stage (MS-2000, Applied scientific instruments, Eugene, OR) was noted and an overview image taken. This process was repeated several times for different positions. After $30 \mathrm{~min}$ the micrometer stage was moved back to the first position. The position was confirmed by recording coarse overview images of the dendrite and thereby adjusting the z-position to the right depth. STED images were recorded as z-stacks of $\sim 20-40 \mu \mathrm{m}$ in $\mathrm{x}$ and $\mathrm{y}$ with $500 \mathrm{~nm}$ axial steps at different positions of the cranial window. All positions were repeatedly imaged 3-4 times at intervals of $30 \mathrm{~min}$ to 2 hours. Therefore, some dendritic regions were investigated at the time points $0 \mathrm{~min}, 30 \mathrm{~min}$ and $60 \mathrm{~min}$, while other dendritic region within the cranial window were examined at the time points $0 \mathrm{~min}, 60 \mathrm{~min}$ and $120 \mathrm{~min}$ or even at $0 \mathrm{~min}, 120 \mathrm{~min}$ and $240 \mathrm{~min}$. The benefit of a membrane label over the often used volume label is that the brightness of the spines and dendrites is similar whereas with a volume label spine heads and dendrites often outshine the small spine neck; thus, either the much darker neck is not visible between the bright head and dendrite or the detector is saturated at the bright heads and dendrites.

All images were recorded with a pixel dwell time of $5 \mu \mathrm{s}$, a pixel size of $30 \times 30 \mathrm{~nm}$ in $x$ and $y$, and zstacks of $500 \mathrm{~nm}$ step size. Blue and green excitation power was $4.5 \mu \mathrm{W}$, respectively, in the back aperture of the objective. The average STED power in the back aperture was $37-45 \mathrm{~mW}$ for static images and $15 \mathrm{~mW}$ for time-lapse imaging.

\section{Data processing and analysis}

Confocal and STED images were acquired by the software Imspector (Abberior Instruments, Göttingen, Germany). Analysis of size, shape, and brightness was performed manually in Fiji ${ }^{55}$. For spine head and PSD95 analysis, the first and last plane were omitted to ensure that the spine (or PSD95, respectively) was located completely in the focal plane. We only analyzed spines that also bore a PSD and we only analyzed spines that extended from the dendrite parallel to the focal plane. PSDs directly on the dendrite most likely representing shaft synapses and spines pointing upwards or downwards that could not be clearly resolved were not analyzed. First, images of each channel were processed as follows (Fiji commands shown in capital letters): 1. Smoothing: PROCESS > SMOOTH once. 2. Brightness adjustment: IMAGE > ADJUST > BRIGHTNESS/CONTRAST set minimum to 1 instead of zero. 3. Overlay both one-color images: IMAGE > COLOR > MERGE CHANNELS. 4. Open ROI manager: ANALYSE > TOOLS $>$ ROI MANAGER. To determine the length $(a)$ and width $(b)$ of PSD95 and the spine head, a line from the top to the bottom, or from left to right as shown in Fig. 2A was drawn (from tip to tip of the corresponding arrowheads) and saved to the ROI manager. For PSD95 assemblies showing a perforation such as shown in Fig. $2 \mathrm{~A}$, we took the maximal length and width to cover the whole assembly. From these values, the area $(A)$ was estimated by computing the area of an ellipse by $A=\pi^{*} a / 2^{*} b / 2^{*}$. For the analysis of brightness, PSD95 images were processed as described above. Using the ELLIPTICAL SELECTION tool, each PSD95 assembly was encircled and the brightness was displayed by the variable "RawintDen", which is the sum of the intensity values of all pixels in the selected area. Spine density was obtained by dividing the total number of spines minus one by the length of the parent dendrite between the first and last spine. The length was measured with the FREEHAND LINE tool, in Z PROJECTION (maximum intensity) images. Branched spines were counted once.

Absolute changes of spine head area or PSD95 area between two time points, $t$ and $t+1$, were calculated by $\Delta A_{a b s}=A(t+1)-A(t)$. ' $A$ ' denotes the spine head area or the PSD area, respectively. Normalized relative changes of spine head area or PSD area were computed by $\Delta A_{\text {norm }}=(A(t+1)$ $A(t)) /(A(t+1)+A(t))$ and percentage changes by $\Delta A_{\%}=(A(t+1)-A(t)) / A(t) * 100$.

Data were further analyzed with MATLAB $^{\circledR}$ (MathWorks ${ }^{\circledR}$, Natick, MA). The principal component analysis (PCA) was performed with the built in MATLAB function 'princomp'. 
The correlation function was computed for different time intervals $\Delta t$ by

$\operatorname{Corr}(\Delta t)=<\delta A 1(t+\Delta t)^{*} \delta A 2(t)>/ \operatorname{sqr} t\left(<\delta A 1(t+\Delta t)^{2}>*<\delta A 2(t)^{2}>\right)$,

with $\delta$ denoting the fluctuation of the mean: $\delta A(t)=A(t)-<A(t)>$. The bracket $<\ldots>$ denotes the average over time and over the empirical distribution of spines or PSDs, respectively. The auto-correlation was computed with $\mathrm{A} 1=\mathrm{A} 2$ either for spine head area or PSD95 area. The cross-correlation was computed with $\mathrm{A} 1$ denoting the spine head area and A2 the PSD95 area.

\section{Nanoorganization and morphological changes of PSD95}

All non-macular PSD95 nanoorganizations were selected. Their shape was categorized by 3 blinded scientists into perforated or clustered shapes. Perforated PSDs were of continuous shape but complex such as a ring or horseshoe-like or more twisted. Clustered nanoorganizations were characterized by two or more separated assemblies of PSD95 per spine and the number of clusters was counted. Temporal changes in PSD95 morphology were analyzed similarly. Assemblies which did not alter their overall morphology or number of clusters were marked as 'no change'. Assemblies showing minor modifications, such as a small movement of a sub-cluster, were classified as 'subtle change'. Changes of the overall morphology such as a smooth continuous PSD falling apart into different clusters were characterized as 'strong change'. All changes were referred to time point $t=0 \mathrm{~min}$.

\section{Statistical analysis}

Statistical analysis was performed in GraphPad Prism (version 7.04, Graphpad Software, San Diego, CA). Positively skewed data sets were compared by the Kolmogorov Smirnov test. Log-transformed data and normalized relative changes were normally distributed and compared by an unpaired $t$-test with Welch's correction. The number of analysed mice and spines, $p$-value, and the specific statistical test performed for each experiment are included in the appropriate figure legend. All tests were applied two-sided where applicable. Probabilities are symbolized by asterisks as follows: ${ }^{*} p<0.05 ;{ }^{* *} p$ $<0.01 ; * * \mathrm{p}<0.001, * * * * \mathrm{p}<0.0001$

\section{Materials availability}

This study did not generate new unique reagents.

\section{Data and materials availability}

Image data files are provided by reasonable request through the corresponding author.

\section{Acknowledgments}

We thank Dr. S. Löwel (Göttingen University) for suggesting and providing the Marlau ${ }^{\text {TM }}$ cages, the animal facility of the MPI for Experimental Medicine for excellent support and Dr. Karl Deisseroth and Dr. Don Arnold for providing plasmids. This work was funded by the Deutsche Forschungsgemeinschaft (DFG, German Research Foundation) within the DFG Research Center and Cluster of Excellence (EXC 171, Area A1) "Nanoscale Microscopy and Molecular Physiology of the Brain" (WW, HS, KIW) and under Germany's Excellence Strategy - EXC 2067/1- 390729940 (KIW).

\section{Author contributions}

Conceptualization (WW, KIW); STED microscope design and construction (KIW); Cloning and virus production (WW, CG); Surgery (HS, WW); Imaging (WW, KIW); Analysis (WW, KIW, FW); Writing (WW, KIW).

\section{Declaration of interest}

The authors declare no competing interests. 


\section{References}

1. Mandolesi, L. et al. Environmental Factors Promoting Neural Plasticity: Insights from Animal and Human Studies. Neural Plast. 2017, 7219461 (2017).

2. Nithianantharajah, J. \& Hannan, A. J. Enriched environments, experience-dependent plasticity and disorders of the nervous system. Nat. Rev. Neurosci. 7, 697-709 (2006).

3. Ziv, N. E. \& Brenner, N. Synaptic Tenacity or Lack Thereof: Spontaneous Remodeling of Synapses. Trends Neurosci. 41, 89-99 (2018).

4. Mongillo, G., Rumpel, S. \& Loewenstein, Y. Intrinsic volatility of synaptic connections - a challenge to the synaptic trace theory of memory. Curr. Opin. Neurobiol. 46, 7-13 (2017).

5. Sigler, A. et al. Formation and Maintenance of Functional Spines in the Absence of Presynaptic Glutamate Release. Neuron 94, 304-311.e4 (2017).

6. Hazan, L. \& Ziv, N. E. Activity Dependent and Independent Determinants of Synaptic Size Diversity. J. Neurosci. 40, 2828-2848 (2020).

7. Yuste, R. \& Bonhoeffer, T. Morphological Changes in Dendritic Spines Associated with LongTerm Synaptic Plasticity. Annu. Rev. Neurosci. 24, 1071-1089 (2001).

8. Chazeau, A. \& Giannone, G. Organization and dynamics of the actin cytoskeleton during dendritic spine morphological remodeling. Cell. Mol. Life Sci. 73, 3053-3073 (2016).

9. Lee, K. F. H., Soares, C. \& Béïque, J. C. Examining form and function of dendritic spines. Neural Plast. 2012, (2012).

10. Herring, B. E. \& Nicoll, R. A. Long-Term Potentiation: From CaMKII to AMPA Receptor Trafficking. Annu. Rev. Physiol. 78, 351-365 (2016).

11. Compans, B., Choquet, D. \& Hosy, E. Review on the role of AMPA receptor nano-organization and dynamic in the properties of synaptic transmission. Neurophotonics 3, 041811 (2016).

12. Matsuzaki, M., Honkura, N., Ellis-Davies, G. C. R. \& Kasai, H. Structural basis of long-term potentiation in single dendritic spines. Nature 429, 761-766 (2004).

13. Bosch, M. et al. Structural and molecular remodeling of dendritic spine substructures during long-term potentiation. Neuron 82, 444-459 (2014).

14. Meyer, D., Bonhoeffer, T. \& Scheuss, V. Balance and Stability of Synaptic Structures during Synaptic Plasticity. Neuron 82, 430-443 (2014).

15. Arellano, J. I., Benavides-Piccione, R., Defelipe, J. \& Yuste, R. Ultrastructure of dendritic spines: correlation between synaptic and spine morphologies. Front. Neurosci. 1, 131-43 (2007).

16. Cane, M., Maco, B., Knott, G. \& Holtmaat, A. The Relationship between PSD-95 Clustering and Spine Stability In Vivo. J. Neurosci. 34, 2075-2086 (2014).

17. Harris, K. M., Jensen, F. E. \& Tsao, B. Three-dimensional structure of dendritic spines and synapses in rat hippocampus (CA1) at postnatal day 15 and adult ages: implications for the maturation of synaptic physiology and long-term potentiation. J. Neurosci. 12, 2685-705 (1992).

18. Leggio, M. G. et al. Environmental enrichment promotes improved spatial abilities and enhanced dendritic growth in the rat. Behav. Brain Res. 163, 78-90 (2005).

19. Gelfo, F., De Bartolo, P., Giovine, A., Petrosini, L. \& Leggio, M. G. Layer and regional effects of 
environmental enrichment on the pyramidal neuron morphology of the rat. Neurobiol. Learn. Mem. 91, 353-365 (2009).

20. Nithianantharajah, J., Levis, H. \& Murphy, M. Environmental enrichment results in cortical and subcortical changes in levels of synaptophysin and PSD-95 proteins. Neurobiol. Learn. Mem. 81, 200-10 (2004).

21. Tønnesen, J., Nadrigny, F., Willig, K. I., Wedlich-Söldner, R. \& Nägerl, U. V. Two-Color STED Microscopy of Living Synapses Using A Single Laser-Beam Pair. Biophys. J. 101, 2545-2552 (2011).

22. Loewenstein, Y., Kuras, A. \& Rumpel, S. Multiplicative dynamics underlie the emergence of the log-normal distribution of spine sizes in the neocortex in vivo. J. Neurosci. 31, 9481-8 (2011).

23. Hofer, S. B., Mrsic-Flogel, T. D., Bonhoeffer, T. \& Hübener, M. Experience leaves a lasting structural trace in cortical circuits. Nature 457, 313-7 (2009).

24. Chen, J. L. et al. Clustered Dynamics of Inhibitory Synapses and Dendritic Spines in the Adult Neocortex. Neuron 74, 361-373 (2012).

25. Stewart, M. G. et al. Chemically induced long-term potentiation increases the number of perforated and complex postsynaptic densities but does not alter dendritic spine volume in CA1 of adult mouse hippocampal slices. Eur. J. Neurosci. 21, 3368-3378 (2005).

26. Broadhead, M. J. et al. PSD95 nanoclusters are postsynaptic building blocks in hippocampus circuits. Sci. Rep. 6, 24626 (2016).

27. Nair, D. et al. Super-resolution imaging reveals that AMPA receptors inside synapses are dynamically organized in nanodomains regulated by PSD95. J. Neurosci. 33, 13204-24 (2013).

28. MacGillavry, H. D., Song, Y., Raghavachari, S. \& Blanpied, T. A. Nanoscale scaffolding domains within the postsynaptic density concentrate synaptic ampa receptors. Neuron 78, 615-622 (2013).

29. Fukata, Y. et al. Local palmitoylation cycles define activity-regulated postsynaptic subdomains. J. Cell Biol. 202, 145-161 (2013).

30. Wegner, W., Mott, A. C., Grant, S. G. N., Steffens, H. \& Willig, K. I. In vivo STED microscopy visualizes PSD95 sub-structures and morphological changes over several hours in the mouse visual cortex. Sci. Rep. 8, 219 (2018).

31. Gross, G. G. et al. Recombinant Probes for Visualizing Endogenous Synaptic Proteins in Living Neurons. Neuron 78, 971-985 (2013).

32. Baroncelli, L. et al. Experience-dependent reactivation of ocular dominance plasticity in the adult visual cortex. Exp. Neurol. 226, 100-109 (2010).

33. Kalogeraki, E., Pielecka-Fortuna, J. \& Löwel, S. Environmental enrichment accelerates ocular dominance plasticity in mouse visual cortex whereas transfer to standard cages resulted in a rapid loss of increased plasticity. PLoS One 12, 1-24 (2017).

34. Willig, K. I. et al. Nanoscopy of filamentous actin in cortical dendrites of a living mouse. Biophys. J. 106, (2014).

35. Eggeling, C., Willig, K. I., Sahl, S. J. \& Hell, S. W. Lens-based fluorescence nanoscopy. Q Rev Biophys 48, 178-243 (2015). 
36. Willig, K. I. et al. Nanoscale resolution in GFP-based microscopy. Nat. Methods 3, 721-723 (2006).

37. Nagerl, U. V., Willig, K. I., Hein, B., Hell, S. W. \& Bonhoeffer, T. Live-cell imaging of dendritic spines by STED microscopy. Proc. Natl. Acad. Sci. 105, 18982-18987 (2008).

38. Kameda, H. et al. Targeting green fluorescent protein to dendritic membrane in central neurons. Neurosci. Res. 61, 79-91 (2008).

39. Steffens, H., Wegner, W. \& Willig, K. I. In vivo STED microscopy: A roadmap to nanoscale imaging in the living mouse. Methods 174, 42-48 (2020).

40. Harris, K. M. \& Weinberg, R. J. Ultrastructure of Synapses in the Mammalian Brain. Cold Spring Harb. Perspect. Biol. 4, a005587-a005587 (2012).

41. Statman, A., Kaufman, M., Minerbi, A., Ziv, N. E. \& Brenner, N. Synaptic Size Dynamics as an Effectively Stochastic Process. PLoS Comput. Biol. 10, (2014).

42. Artola, A. et al. Long-lasting modulation of the induction of LTD and LTP in rat hippocampal CA1 by behavioural stress and environmental enrichment. Eur. J. Neurosci. 23, 261-272 (2006).

43. Villa, K. L. et al. Inhibitory Synapses Are Repeatedly Assembled and Removed at Persistent Sites In Vivo. Neuron 89, 756-769 (2016).

44. Moneron, G. \& Hell, S. W. Two-photon excitation STED microscopy. Opt. Express 17, 14567 (2009).

45. Panatier, A., Arizono, M. \& Nagerl, U. V. Dissecting tripartite synapses with STED microscopy. Philos. Trans. R. Soc. B Biol. Sci. 369, 20130597-20130597 (2014).

46. Stein, V., House, D. R. C., Bredt, D. S. \& Nicoll, R. A. Postsynaptic density-95 mimics and occludes hippocampal long-term potentiation and enhances long-term depression. J. Neurosci. 23, 5503-6 (2003).

47. El-Husseini, A., Schnell, E. \& Chetkovich, D. PSD-95 involvement in maturation of excitatory synapses. Science 290, 1364-8 (2000).

48. Cook, S. G., Goodell, D. J., Restrepo, S., Arnold, D. B. \& Bayer, K. U. Simultaneous Live Imaging of Multiple Endogenous Proteins Reveals a Mechanism for Alzheimer's-Related Plasticity Impairment. Cell Rep. 27, 658-665.e4 (2019).

49. Lee, H. K. \& Kirkwood, A. Mechanisms of Homeostatic Synaptic Plasticity in vivo. Front. Cell. Neurosci. 13, 1-7 (2019).

50. Chen, H., Tang, A. H. \& Blanpied, T. A. Subsynaptic spatial organization as a regulator of synaptic strength and plasticity. Curr. Opin. Neurobiol. 51, 147-153 (2018).

51. Buschler, A. \& Manahan-Vaughan, D. Brief environmental enrichment elicits metaplasticity of hippocampal synaptic potentiation in vivo. Front. Behav. Neurosci. 6, 1-10 (2012).

52. Wegner, W. et al. In vivo mouse and live cell STED microscopy of neuronal actin plasticity using far-red emitting fluorescent proteins. Sci. Rep. 7, 11781 (2017).

53. Fares, R. P., Kouchi, H. \& Bezin, L. Standardized environmental enrichment for rodents in Marlau cage. Protoc. Exch. 1-14 (2012). doi:10.1038/protex.2012.036

54. D'Este, E., Kamin, D., Balzarotti, F. \& Hell, S. W. Ultrastructural anatomy of nodes of Ranvier in the peripheral nervous system as revealed by STED microscopy. Proc. Natl. Acad. Sci. 114, 
bioRxiv preprint doi: https://doi.org/10.1101/2020.10.23.352195; this version posted October 23, 2020. The copyright holder for this preprint (which was not certified by peer review) is the author/funder, who has granted bioRxiv a license to display the preprint in perpetuity. It is made available under aCC-BY-NC-ND 4.0 International license.

E191-E199 (2017).

55. Schindelin, J. et al. Fiji: an open-source platform for biological-image analysis. Nat. Methods $\mathbf{9}$, 676-682 (2012). 